\title{
Pediatric Pituitary Adenoma: Case Series, Review of the Literature, and a Skull Base Treatment Paradigm
}

\author{
Avital Perry ${ }^{1}$ Christopher Salvatore Graffeo ${ }^{1} \quad$ Christopher Marcellino $^{1} \quad$ Bruce E. Pollock $^{1}$ \\ Nicholas M. Wetjen ${ }^{1}$ Fredric B. Meyer ${ }^{1}$ \\ ${ }^{1}$ Department of Neurologic Surgery, Mayo Clinic, Rochester \\ Minnesota, United States \\ Address for correspondence Fredric B. Meyer, MD, Department of \\ Neurologic Surgery - Mayo Clinic, 200 First St SW, Rochester, MN \\ 55905, United States (e-mail: Meyer.Fredric@mayo.edu).
}

J Neurol Surg B 2018;79:91-114.

\begin{abstract}
Keywords

- pediatric pituitary adenoma

- transsphenoidal surgery

- radiotherapy

- stereotactic radiosurgery

- hypopituitarism

Background Pediatric pituitary adenoma is a rare skull base neoplasm, accounting for $3 \%$ of all intracranial neoplasms in children and $5 \%$ of pituitary adenomas. Compared with pituitary tumors in adults, secreting tumors predominate and longer disease trajectories are expected due to the patient age resulting in a natural history and treatment paradigm that is complex and controversial.

Objectives The aims of this study were to describe a large, single-institution series of pediatric pituitary adenomas with extensive long-term follow-up and to conduct a systematic review examining outcomes after pituitary adenoma surgery in the pediatric population.

Methods The study cohort was compiled by searching institutional pathology and operative reports using diagnosis and site codes for pituitary and sellar pathology, from 1956 to 2016. Systematic review of the English language literature since 1970 was conducted using PubMed, MEDLINE, Embase, and Google Scholar.

Results Thirty-nine surgically managed pediatric pituitary adenomas were identified, including 15 prolactinomas, 14 corticotrophs, 7 somatotrophs, and 4 non-secreting adenomas. All patients underwent transsphenoidal resection (TSR) as the initial surgical treatment. Surgical cure was achieved in 18 (46\%); 21 experienced recurrent/persistent disease, with secondary treatments including repeat surgery in 10 , radiation in 14 , adjuvant pharmacotherapy in 11 , and bilateral adrenalectomy in 3 . At the last follow-up (median 87 months, range 3-581), nine remained with recurrent/ persistent disease (23\%).

Thirty-seven publications reporting surgical series of pediatric pituitary adenomas were included, containing 1,284 patients. Adrenocorticotropic hormone (ACTH)secreting tumors were most prevalent (43\%), followed by prolactin (PRL)-secreting (37\%), growth hormone (GH)-secreting (12\%), and nonsecreting (7\%). Surgical cure was reported in $65 \%$. Complications included pituitary insufficiency (23\%), permanent visual dysfunction (6\%), chronic diabetes insipidus (DI) (3\%), and postoperative cerebrospinal fluid (CSF) leak (4\%). Mean follow-up was 63 months (range 0-240), with recurrent/persistent disease reported in $18 \%$ at the time of last follow-up.
\end{abstract}

published online January 24, 2018
(C) 2018 Georg Thieme Verlag KG Stuttgart · New York
DOI https://doi.org/ $10.1055 / \mathrm{s}-0038-1625984$. ISSN 2193-6331. 
Conclusion Pediatric pituitary adenomas are diverse and challenging tumors with complexities far beyond those encountered in the management of routine adult pituitary disease, including nuanced decision-making, a technically demanding operative environment, high propensity for recurrence, and the potentially serious consequences of hypopituitarism with respect to fertility and growth potential in a pediatric population. Optimal treatment requires a high degree of individualization, and patients are most likely to benefit from consolidated, multidisciplinary care in highly experienced centers.

\section{Introduction}

Pediatric pituitary adenoma is a rare disease, representing $3 \%$ of all intracranial neoplasms in children, and $~ 5 \%$ of all pituitary adenomas. ${ }^{1-5}$ As compared with the adult disease, pituitary adenoma in children is predominantly comprised of secreting tumors, with prolactin (PRL), adrenocorticotropic hormone (ACTH), and growth hormone $(\mathrm{GH})$ secreting tumors observed most frequently. ${ }^{4-11}$ This contrast is most likely attributable to the slow progression of non-secreting tumors, which theoretically may not grow sufficiently in early life to induce symptoms. A combination of advances in our understanding of the underlying disease; on-going developments in radiation and endoscopic technology, and techniques; and shifting attitudes regarding the goals-ofcare have cumulatively resulted in a highly nuanced clinical landscape.

Due to the combined rarity and complexity of the disease, pediatric pituitary adenoma has been infrequently studied, and recommendations regarding its optimal management are disparate, debated, and based on relatively poor evidence. Correspondingly, our objective was to report our own experience with these challenging tumors, systematically review the preceding literature, and assemble our findings into a treatment algorithm salient to the clinical practice of pediatric skull base surgery.

\section{Methods}

\section{Patient Search, Inclusion Criteria, and Clinical Endpoints}

The study cohort was compiled by searching institutional pathology and operative reports using diagnosis and site codes for pituitary and sellar pathology, from 1956 to 2016; positive results in patients aged 25 years and younger at time of treatment were cross-referenced with operative reports and surgical databases to confirm that patients underwent neurosurgical treatment at our institution for pituitary adenoma. Patients over 18 years at the time of diagnosis were excluded. Included patients underwent retrospective chart review to capture relevant clinical outcomes ( - Tables 1-3). Given the complexities of pituitary adenoma care and the challenges of definitively identifying periods of true disease remission, in our series and review of the literature, we grouped all failures of primary surgical therapy as a single entity we refer to as recurrent or persistent disease,
Table 1 Overview of the study cohort

\begin{tabular}{|c|c|}
\hline & $n=39$ \\
\hline $\begin{array}{l}\text { Age at time of diagnosis } \\
\text { (years) }\end{array}$ & $15(8-18)$ \\
\hline $\begin{array}{l}\text { Age at time of first operation } \\
\text { (years) }\end{array}$ & $16(9-22)$ \\
\hline \multicolumn{2}{|c|}{ General neurologic symptoms or focal deficits } \\
\hline Headache & $26(67 \%)$ \\
\hline Visual disturbance & $14(36 \%)$ \\
\hline Cranial neuropathy & $5(13 \%)$ \\
\hline Depression & $5(13 \%)$ \\
\hline Seizure & $2(5 \%)$ \\
\hline Diplopia & $1(3 \%)$ \\
\hline Stroke & $1(3 \%)$ \\
\hline Vertigo & $1(3 \%)$ \\
\hline \multicolumn{2}{|c|}{ Nonspecific symptoms of pituitary dysfunction } \\
\hline Arrested growth & $6(15 \%)$ \\
\hline Hypothyroidism & $6(15 \%)$ \\
\hline Apoplexy & $4(10 \%)$ \\
\hline Pubertal delay & $3(8 \%)$ \\
\hline Polyuria & $1(3 \%)$ \\
\hline \multicolumn{2}{|c|}{ Symptoms suggesting hyperprolactinemia } \\
\hline Amenorrhea $^{a}$ & $11(28 \%)$ \\
\hline Galactorrhea & $7(18 \%)$ \\
\hline \multicolumn{2}{|c|}{ Symptoms suggesting hypercortisolemia } \\
\hline Obesity/weight gain & $16(41 \%)$ \\
\hline Acne & $12(31 \%)$ \\
\hline Hirsutism & $11(28 \%)$ \\
\hline Moon facies & $10(26 \%)$ \\
\hline Striae & $6(15 \%)$ \\
\hline Buffalo hump & $5(13 \%)$ \\
\hline Easy bruising & $5(13 \%)$ \\
\hline Muscle weakness & $4(10 \%)$ \\
\hline Acanthosis nigricans & $2(5 \%)$ \\
\hline Pathologic fracture & $1(3 \%)$ \\
\hline
\end{tabular}


Table 1 (Continued)

\begin{tabular}{|c|c|}
\hline & $n=39$ \\
\hline \multicolumn{2}{|c|}{ Symptoms suggesting hypersomatotropinemia } \\
\hline Precocious growth & $5(13 \%)$ \\
\hline Acromegaly/gigantism & $4(10 \%)$ \\
\hline $\begin{array}{l}\text { Maximum tumor diameter on pre- } \\
\text { operative imaging ( } \mathrm{mm} \text {; median } \\
\text { (range)) }\end{array}$ & $11(1-40)$ \\
\hline \multicolumn{2}{|l|}{ Biochemical and pathologic diagnosis } \\
\hline Prolactin secreting & $15(39 \%)$ \\
\hline ACTH secreting & $14(36 \%)$ \\
\hline GH secreting & $7(18 \%)$ \\
\hline Non-secreting & $6(15 \%)$ \\
\hline Pluri-hormonal & $4(10 \%)$ \\
\hline Atypical features & $5(13 \%)$ \\
\hline Crooke's hyaline change & $4(10 \%)^{b}$ \\
\hline \multicolumn{2}{|l|}{ Underlying genetic conditions } \\
\hline Multiple endocrine neoplasia type 1 & $1(3 \%)$ \\
\hline McCune-Albright syndrome & $1(3 \%)$ \\
\hline
\end{tabular}

Abbreviations: ACTH; adrenocorticotropic hormone; $\mathrm{GH}$, growth hormone.

a Percentage of female patients only.

bercentage of ACTH-secreting adenomas.

which we defined as any symptomatic, biochemical, or radiographic evidence of disease at any time following the first operation. Among patients who were identified as having recurrent or persistent disease, we documented disease cure only where explicit evidence confirmed that a patient was symptom-free, off tumor-suppressive pharmacotherapy, and with resolution of any previously documented biochemical and/or radiographic disease.

\section{Systematic Review}

A search of the English language literature since 1970 was conducted using PubMed, MEDLINE, Embase, and Google Scholar. Keywords and MeSH terms included "pituitar*" or "hypophys*" in combination with "child*," "pediatr*," "paediatr*," or "adolesc" and "adenoma” (-Fig. 1). Initial results after deduplication yielded 57 unique English language publications; bibliographies were screened for additional references potentially warranting inclusion, and all abstracts were independently reviewed by two authors to confirm that inclusion criteria were met (defined as case series of biochemically, radiographically, or pathologically confirmed pituitary adenoma reporting extractable treatment and outcomes data); instances of disagreement were secondarily re-reviewed and discussed for final adjudication. Thirty-seven eligible publications were identified, 11 of which reported patients treated up to 20 years-of-age, rather than diagnosed up to 18 years-of-age, which were deemed a comparable population and included to maximize
Table 2 Surgical management and outcomes

\begin{tabular}{|l|l|}
\hline & $n=39$ \\
\hline $\begin{array}{l}\text { History of preoperative } \\
\text { pharmacotherapy }\end{array}$ & $13(33 \%)$ \\
\hline Microscopic TSR & $37(95 \%)$ \\
\hline Endoscopic endonasal TSR & $2(5 \%)$ \\
\hline Gross total resection & $18(46 \%)$ \\
\hline Disease cured with TSR alone & $18(46 \%)$ \\
\hline $\begin{array}{l}\text { Recurrent/persistent disease after } \\
\text { initial TSR }\end{array}$ & $21(54 \%)$ \\
\hline Any repeat operation & $10(26 \%)$ \\
\hline Any postoperative radiation & $14(36 \%)$ \\
\hline $\begin{array}{l}\text { Any postoperative } \\
\text { pharmacotherapy }\end{array}$ & $11(28 \%)$ \\
\hline Bilateral adrenalectomy & $3(21 \%)^{\mathrm{a}}$ \\
\hline Treatment complications & $26(67 \%)$ \\
\hline $\begin{array}{l}\text { Chronic postoperative pituitary } \\
\text { insufficiency }\end{array}$ & $3(8 \%)$ \\
\hline Postoperative CSF leak & $1(3 \%)$ \\
\hline $\begin{array}{l}\text { Permanent postoperative visual } \\
\text { dysfunction }\end{array}$ & $1(3 \%)$ \\
\hline Chronic diabetes insipidus & $1(3 \%)$ \\
\hline Radiation necrosis & $1(3 \%)$ \\
\hline Radiation-induced optic neuropathy \\
\hline Radiation-induced abducens palsy \\
\hline Meningitis & $97(3-581)$ \\
\hline $\begin{array}{l}\text { Total clinical follow-up (mo.; median } \\
\text { follow-up }\end{array}$ & $13 \%)$ \\
\hline Mortalities & 1 ) \\
\hline
\end{tabular}

Abbreviations: ACTH, adrenocorticotropic hormone; TSR, transsphenoidal resection.

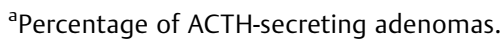

yield. All 37 publications were reviewed in detail; relevant clinical outcomes were again captured (-Tables 4-5).

\section{Results}

\section{Overview of the Study Cohort}

Thirty-nine pediatric pituitary adenoma patients at our institution were identified; median ages at times of diagnosis and surgery were 15 and 16 years, respectively (ranges 8-18 and 9-22, respectively). Symptoms at the time of presentation were diverse and heterogeneous, with the most common complaints including headache (67\%), obesity/weight gain (41\%), visual disturbance (36\%), acne (31\%), amenorrhea (28\%), hirsutism (28\%), and moon facies (26\%; - Table 1). Median maximum tumor diameter on preoperative imaging was $11 \mathrm{~mm}$ (range 1-40 mm). Among 39 adenomas, biochemical and pathologic analyses diagnosed 15 prolactinomas 
Table 3 Detailed treatment courses in recurrent or persistent disease

\begin{tabular}{|l|l|}
\hline $\begin{array}{l}\text { Successfully treated recurrent/persistent } \\
\text { tumors (after failed primary TSR) }\end{array}$ & $\mathbf{n = 2 1 ^ { \text { a } }}$ \\
\hline Repeat TSR alone, cured at last follow-up & $3(14 \%)$ \\
\hline $\begin{array}{l}\text { SRS alone (one patient underwent two } \\
\text { treatments), cured at last follow-up }\end{array}$ & $4(19 \%)$ \\
\hline $\begin{array}{l}\text { Repeat TSR followed by SRS, cured at last } \\
\text { follow-up }\end{array}$ & $3(14 \%)$ \\
\hline $\begin{array}{l}\text { Repeat TSR followed by PBRT, cured at last } \\
\text { follow-up }\end{array}$ & $1(5 \%)$ \\
\hline $\begin{array}{l}\text { Repeat TSR, BAX, and EBRT, cured at last } \\
\text { follow-up }\end{array}$ & $1(5 \%)$ \\
\hline $\begin{array}{l}\text { Unsuccessfully treated recurrent/persistent } \\
\text { tumors (after failed primary TSR) }\end{array}$ & $9(43 \%)$ \\
\hline $\begin{array}{l}\text { Pharmacotherapy alone, persistent disease } \\
\text { at last follow-up }\end{array}$ & $4(19 \%)$ \\
\hline $\begin{array}{l}\text { EBRT alone, persistent disease at last follow-up } \\
\text { b }\end{array}$ & $1(5 \%)$ \\
\hline $\begin{array}{l}\text { SRS alone, persistent disease at last follow-up } \\
\text { b }\end{array}$ & $1(5 \%)$ \\
\hline $\begin{array}{l}\text { Repeat TSR, BAX, and SRS, persistent disease } \\
\text { at last follow-up }\end{array}$ & $1(5 \%)$ \\
\hline $\begin{array}{l}\text { EBRT, multiple TSRs, and craniotomy, per- } \\
\text { sistent disease at last follow-up }\end{array}$ & $1(5 \%)$ \\
\hline $\begin{array}{l}\text { Multiple TSRs and craniotomies; BAX; mul- } \\
\text { tiplease at last follow-up }\end{array}$ & $1(5 \%)$ \\
\hline
\end{tabular}

Abbreviations: BAX, bilateral adrenalectomy; EBRT, external beam radiotherapy; PBRT, proton beam radiotherapy; SRS, stereotactic radiosurgery; TSR, transsphenoidal resection.

aPercentages of patients with recurrent/persistent disease after first TSR.

batients with atypical features on pathology $(n=5)$.

(39\%), 14 corticotrophs (36\%), 7 somatotrophs (18\%), and 4 non-secreting adenomas (10\%). Four tumors were plurihormonal (10\%): three were positive for PRL and $\mathrm{GH}$ (8\%), and one was positive for $\mathrm{ACTH}$ and $\mathrm{GH}(3 \%)$. Five tumors demonstrated atypical pathologic features (13\%), and four ACTH-secreting tumors contained Crooke's hyaline change (29\%). Underlying genetic conditions were present in one patient with multiple endocrine neoplasia type $1(\mathrm{MEN}-1)$ and one with McCuneAlbright syndrome.

\section{Surgical Management and Outcomes}

An initial trial of at least one pharmacologic agent was attempted in 13 (33\%) patients, typically with bromocriptine or cabergoline, as well as one trial each of pergolide, octreotide, and pegvisomant (-Table 2). Transsphenoidal resection (TSR) was then attempted in 39 (100\%) patients, 37 (95\%) via either a sublabial, transsphenoidal, or transnasal transsphenoidal microsurgical technique and 2 (5\%) using a purely endoscopic endonasal approach (EEA). A primary surgical cure was obtained in 18 patients, in all of whom gross total resection (GTR) was achieved (46\%).

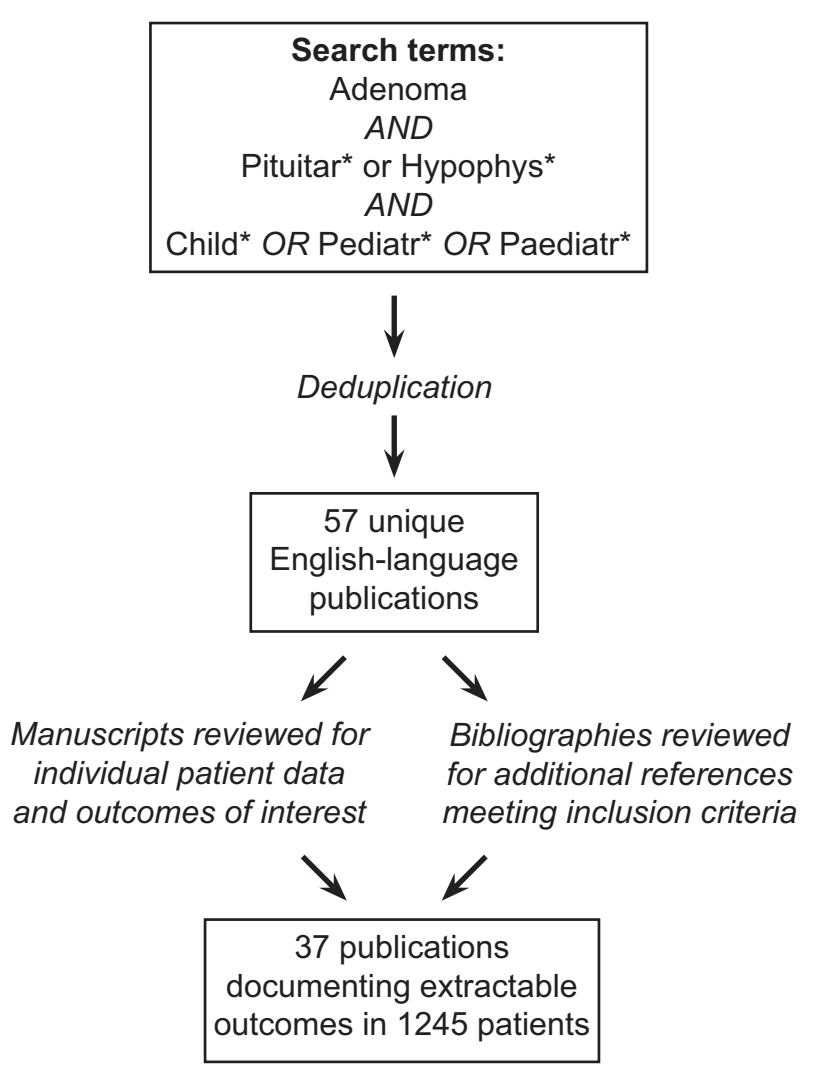

Fig. 1 Schematic depicting search strategy for systematic literature review

Twenty-one patients experienced recurrent or progressive disease postoperatively. Repeat surgery was undertaken in $10(26 \%)$, radiation of any modality was used in 14 (36\%), 11 received pharmacotherapy (28\%), and 3 underwent bilateral adrenalectomy (31\% of ACTH-secreting tumors). Cumulatively, 39 patients underwent a total of 55 TSRs, 7 craniotomies, 13 stereotactic radiosurgeries (SRS), 5 courses of external beam radiotherapy (EBRT), 1 proton beam radiotherapy (PBRT), and 3 bilateral adrenalectomies (BAX). Detailed treatment courses are outlined in -Table 3. Atypical pathologic features were significantly associated with recurrent or persistent disease $(p=0.05)$.

At last clinical follow-up, disease cure had been achieved in $30(77 \%)$ patients including 12 (31\%) who had been treated for recurrent or persistent disease, while $9(25 \%)$ remained with recurrent or persistent disease ( - Table 2). Complications from any treatment included 26 patients with chronic pituitary insufficiency requiring supplementation of at least one hormone $(67 \%)$, three cases $(8 \%)$ of postoperative cerebrospinal fluid (CSF) leak, and one case (3\%) each of permanent visual dysfunction, chronic diabetes insipidus (DI), radiation necrosis, radiation-induced optic neuropathy, radiation-induced abducens palsy, or meningitis. Median total clinical follow up was 87 months (range 3-581). There were no mortalities in our series; however, one patient has initiated palliative care and is anticipated to expire due to primary disease. 


\begin{tabular}{|c|c|c|c|c|c|c|c|c|c|c|c|c|c|c|c|c|c|c|c|c|c|c|c|}
\hline 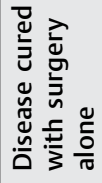 & 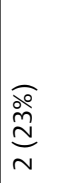 & $\begin{array}{l}\stackrel{\circ}{\stackrel{े}{\circ}} \\
\stackrel{m}{=}\end{array}$ & $\begin{array}{l}\widehat{\curvearrowright} \\
\stackrel{\infty}{\infty} \\
\infty \\
m\end{array}$ & 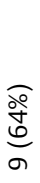 & I & 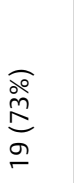 & $\begin{array}{l}\text { o̊ } \\
\text { ᄋे } \\
\text { f }\end{array}$ & 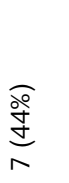 & 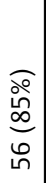 & 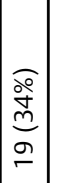 & $\begin{array}{l}\text { ○े } \\
\text { すे } \\
\text { f }\end{array}$ & 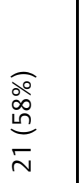 & $\begin{array}{l}\text { ㅇํ } \\
\stackrel{\leftrightarrow}{ \pm} \\
\stackrel{0}{-}\end{array}$ & 1 & $\begin{array}{c} \\
\stackrel{\circ}{\hat{\sigma}} \\
\qquad \\
0\end{array}$ & 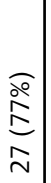 & 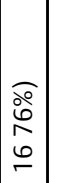 & $\begin{array}{l}\widehat{D} \\
\stackrel{0}{0} \\
\infty \\
\forall\end{array}$ & 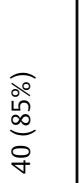 & $\begin{array}{l}\text { ১̊ } \\
\text { 仓 }\end{array}$ & $\begin{array}{l}\frac{20}{\hat{y}} \\
\frac{1}{1}\end{array}$ & 1 & 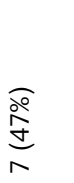 \\
\hline 呂 & 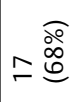 & 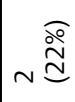 & 1 & 1 & ㅇㅇㅇ & 1 & 1 & 1 & 1 & 1 & 1 & $\approx \stackrel{\widehat{\circ}}{\stackrel{\circ}{\infty}}$ & 1 & 1 & 1 & 1 & 1 & $\begin{array}{r}\widehat{\circ} \\
\stackrel{\infty}{\infty}\end{array}$ & 1 & 1 & 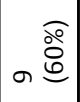 & 1 & 1 \\
\hline 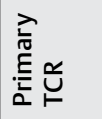 & $\frac{\substack{\stackrel{0}{\infty} \\
\text { d }}}{2}$ & 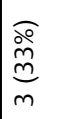 & $\begin{array}{l}\text { @̊ } \\
\text { 仓 }\end{array}$ & 1 & @̊ & 仓̊ㅇํ & $\begin{array}{l}\text { ১े } \\
\stackrel{-}{-}\end{array}$ & 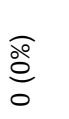 & ஓ̊ & 。ํ. & ஓ̊ㅇㅇ & $\frac{\text { ঃ̊ }}{\stackrel{0}{0}}$ & @̊ & $\frac{\widehat{\circ}}{\stackrel{0}{0}}$ & $\begin{array}{c}0 \\
\stackrel{\circ}{\varrho} \\
0\end{array}$ & 仓̊ & ஓे & 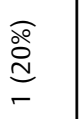 & $\frac{\widehat{\partial}}{\stackrel{\circ}{0}}$ & $\begin{array}{l}\text { ১े } \\
\text { 仓 }\end{array}$ & $\begin{array}{l}\text { ळo } \\
\text { @્ }\end{array}$ & 仓̊ & 1 \\
\hline 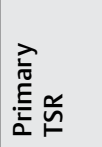 & 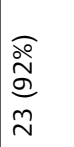 & $\begin{array}{l}\text { ○े } \\
\text { ○े } \\
\text { a }\end{array}$ & 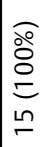 & 1 & 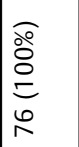 & 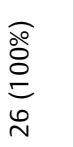 & 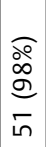 & $\begin{array}{l}\widehat{\circ} \\
\stackrel{0}{0} \\
\stackrel{0}{0}\end{array}$ & $\begin{array}{l}\widehat{\circ} \\
\stackrel{\circ}{\circ} \\
= \\
b \\
\varnothing\end{array}$ & 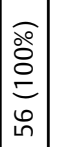 & $\begin{array}{c}\widehat{o} \\
\stackrel{0}{\circ} \\
\stackrel{0}{0} \\
g \\
g\end{array}$ & 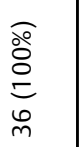 & $\begin{array}{l}\widehat{\circ} \\
\stackrel{\circ}{\circ} \\
\approx \\
\approx\end{array}$ & 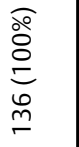 & $\begin{array}{l}\widehat{o} \\
\stackrel{\circ}{\circ} \\
ٍ \\
\sigma\end{array}$ & $\begin{array}{l}\widehat{O} \\
\stackrel{\circ}{0} \\
= \\
\stackrel{n}{n} \\
m\end{array}$ & 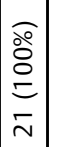 & $\begin{array}{l}\widehat{\partial} \\
\stackrel{0}{0} \\
0 \\
\sigma\end{array}$ & $\begin{array}{l}\frac{\widehat{o}}{\sigma} \\
\frac{m}{y}\end{array}$ & $\begin{array}{l}\stackrel{ㅇ}{\circ} \\
\stackrel{\circ}{\circ}\end{array}$ & 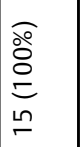 & $\begin{array}{l}\text { } \\
8 \\
0 \\
0 \\
\circ \\
\text { 음 }\end{array}$ & 1 \\
\hline 흔 $\times \frac{\bar{d}}{\bar{\sigma}}$ & 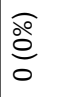 & 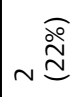 & ஓ̊ & 1 & 1 & $\nabla \stackrel{\widehat{ㅇ ํ ำ ~}}{=}$ & 1 & 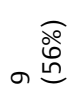 & 1 & $\begin{array}{l}\text { ○े } \\
\text { in } \\
m\end{array}$ & I & 1 & $\begin{array}{l}\stackrel{\circ}{\circ} \\
\stackrel{0}{\circ}\end{array}$ & $=\stackrel{\widehat{\circ}}{\infty}$ & $\begin{array}{c}\stackrel{0}{\circ} \\
\stackrel{0}{0} \\
0\end{array}$ & 1 & $\begin{array}{l}\text { 우 } \\
\text { ஸ் } \\
-\end{array}$ & $\begin{array}{l}\stackrel{0}{\circ} \\
\text { 仓 }\end{array}$ & 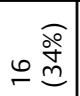 & $m \stackrel{\widehat{ㅇ ํ ํ ~}}{=}$ & @̊ & 1 & $\simeq \stackrel{\bigcirc}{\circ}$ \\
\hline 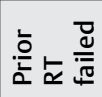 & 仓̊ & $\begin{array}{l}\text { @̊ } \\
\text { 仓 }\end{array}$ & $\begin{array}{l}\text { @̊ } \\
\text { 仓 }\end{array}$ & 1 & 1 & 仓 & 1 & 仓े & 1 & $\begin{array}{l}\text { @ } \\
\stackrel{0}{0} \\
0\end{array}$ & $\begin{array}{l}\text { ঃ } \\
\text { 仓े } \\
0\end{array}$ & 仓े & $\begin{array}{l}\text { ㅇํㄹ } \\
\text { 仓 }\end{array}$ & $\stackrel{\frac{0}{ٍ}}{\stackrel{2}{c}}$ & $\begin{array}{l}\text { @ } \\
\stackrel{0}{0}\end{array}$ & 1 & $\begin{array}{l}\text { ㅇํㄹ } \\
\text { ํํ } \\
-\end{array}$ & 仓 & 仓 & 仓 & 仓 & 1 & 仓̊ \\
\hline 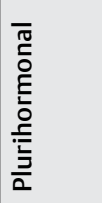 & 1 & $\underset{\substack{\stackrel{D}{d} \\
\stackrel{N}{N}}}{ }$ & $\begin{array}{l}\widehat{\stackrel{\alpha}{0}} \\
\stackrel{m}{m} \\
\text { nn }\end{array}$ & 1 & @̊ & 仓̊ํㅇ & @̊ & $\stackrel{\circ}{\circ}_{0}^{\circ}$ & $\begin{array}{l}\text { ㅇํㅇ } \\
\stackrel{0}{0}\end{array}$ & 1 & $\begin{array}{l}\text { ̊̊ } \\
\stackrel{0}{0}\end{array}$ & $\frac{\substack{\stackrel{\circ}{0}\\
}}{a}$ & $\begin{array}{l}\text { ঃ̊ } \\
\stackrel{0}{0} \\
0\end{array}$ & $\begin{array}{l}\stackrel{\circ}{\stackrel{一}{2}} \\
\text { }\end{array}$ & $\begin{array}{l}\text { ஓ̊ } \\
\stackrel{0}{0} \\
0\end{array}$ & 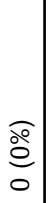 & 㐫 & $\frac{\text { o̊ }}{\stackrel{0}{0}}$ & $\frac{\text { ঃे }}{\stackrel{0}{0}}$ & $\begin{array}{l}\stackrel{0}{\circ} \\
\text { 仓 }\end{array}$ & 仓̊ & $\begin{array}{l}\text { @o } \\
\stackrel{0}{0} \\
\text { on }\end{array}$ & $\begin{array}{l}\text { @̊ } \\
\stackrel{0}{0}\end{array}$ \\
\hline
\end{tabular}

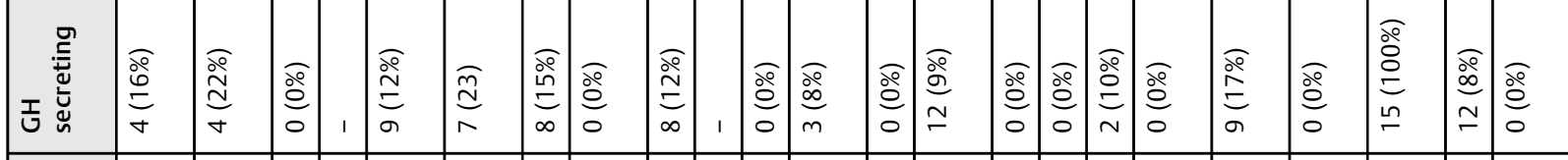

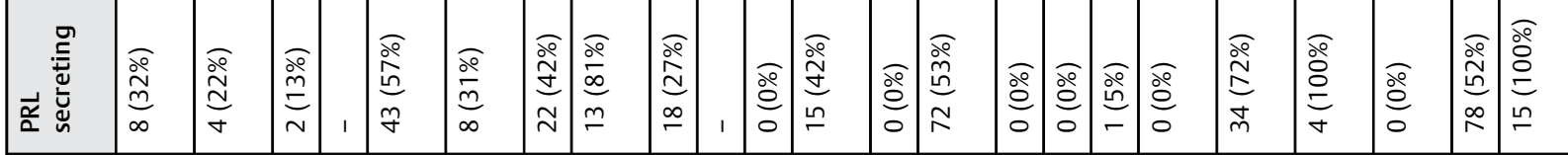

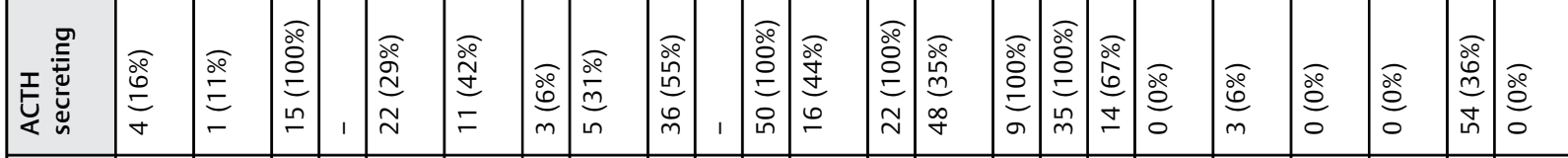

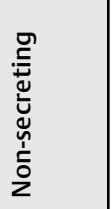

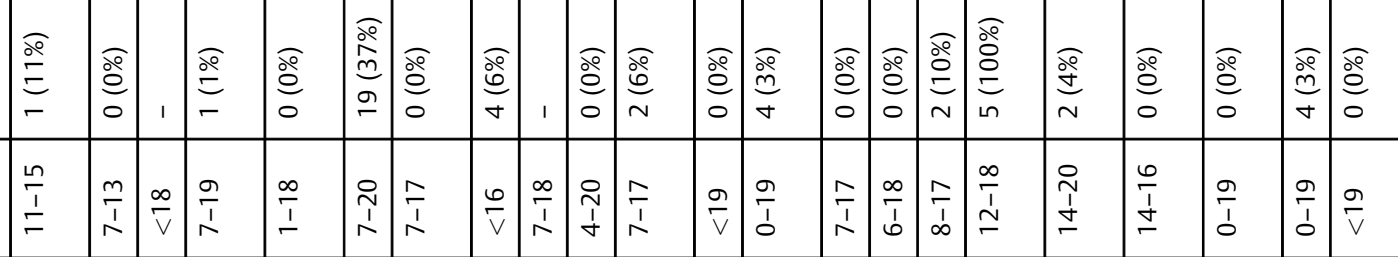

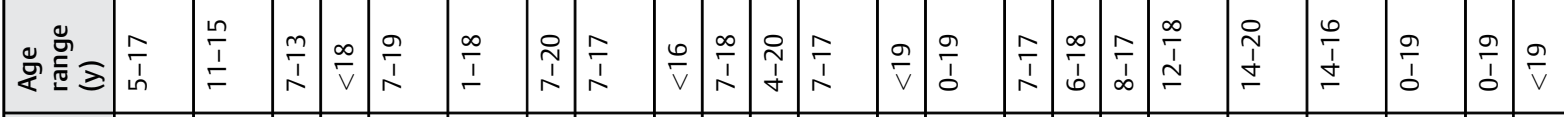

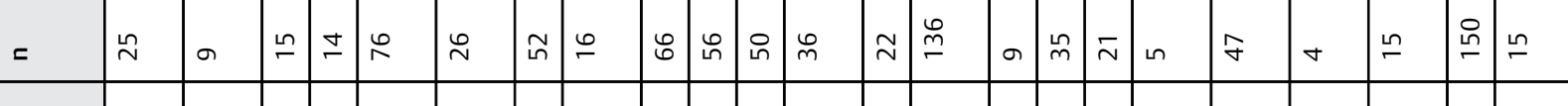

\begin{tabular}{|c|c|c|c|c|c|c|c|c|c|c|c|c|c|c|c|c|c|c|c|c|c|c|c|}
\hline ల్t & $\stackrel{\infty}{\sigma}$ & $\begin{array}{l}\infty \\
\stackrel{\infty}{\sigma} \\
\sigma\end{array}$ & $\begin{array}{l}+ \\
\stackrel{+}{\sigma} \\
\stackrel{\sigma}{\sigma}\end{array}$ & 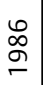 & 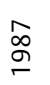 & 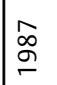 & 各 & হু & ఫু & $\begin{array}{l}\text { g } \\
\sigma\end{array}$ & $\begin{array}{l}\text { S. } \\
\sigma \\
\sigma\end{array}$ & 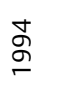 & 㔯 & 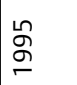 & $\begin{array}{l}\text { 吕 } \\
\sigma\end{array}$ & S. & S̆ & 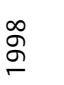 & 各 & $\stackrel{\text { ळ }}{\sigma}$ & gે & g. & 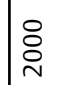 \\
\hline 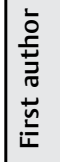 & 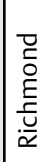 & 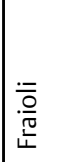 & 䒯 & 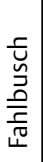 & 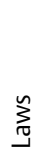 & 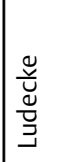 & 营 & $\begin{array}{l}\frac{\pi}{\pi} \\
\frac{\pi}{\pi}\end{array}$ & ठ̀̃ & 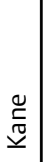 & $\begin{array}{l}\vec{z} \\
\frac{\vec{v}}{0} \\
\overline{0} \\
\bar{n} \\
\Sigma\end{array}$ & 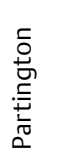 & 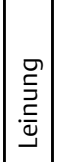 & 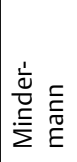 & 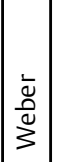 & 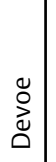 & 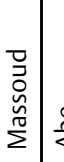 & & 岕 & 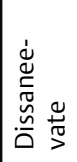 & $\begin{array}{l}\stackrel{0}{<} \\
\text { L }\end{array}$ & & 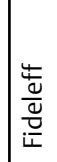 \\
\hline
\end{tabular}




\begin{tabular}{|c|c|c|c|c|c|c|c|c|c|c|c|c|c|c|c|c|}
\hline 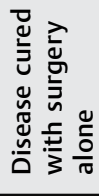 & 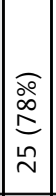 & 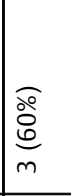 & 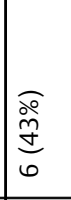 & 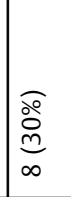 & 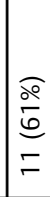 & 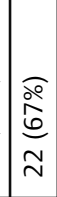 & 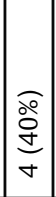 & 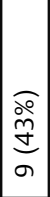 & 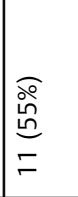 & $\begin{array}{l}\text { ळे } \\
\text { ळे } \\
\mp \\
\mp\end{array}$ & 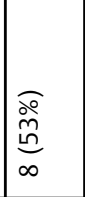 & 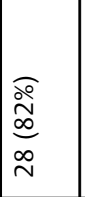 & 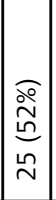 & 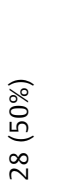 & 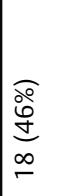 & 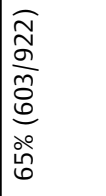 \\
\hline Ĕ & 1 & m & 율 & 1 & 1 & 1 & 1 & 1 & $\simeq$ 氙 & $\simeq \stackrel{\text { वे }}{\circ}$ & m & 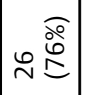 & 1 & 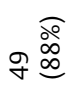 & $\infty \stackrel{\widehat{\circ}}{-\stackrel{8}{d}}$ & 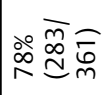 \\
\hline 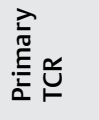 & 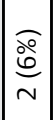 & 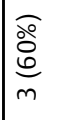 & ठ̊ํํ & 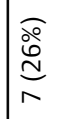 & 总 & 仓̊응 & \begin{tabular}{|c|}
$\stackrel{\circ}{\circ}$ \\
$\stackrel{\circ}{\complement}$ \\
-
\end{tabular} & 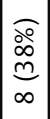 & 产 & 仓̊ & 总 & 仓̊ & @̊ & 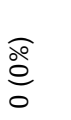 & 总 & 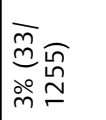 \\
\hline 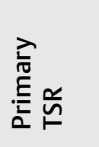 & 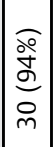 & 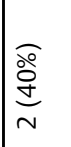 & 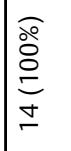 & 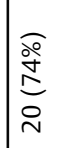 & $\begin{array}{l}\stackrel{\circ}{\circ} \\
\stackrel{0}{\circ} \\
\stackrel{0}{\circ} \\
-\end{array}$ & $\begin{array}{l}\widehat{o} \\
\stackrel{0}{0} \\
\stackrel{0}{c} \\
m \\
m\end{array}$ & 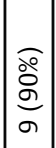 & 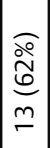 & 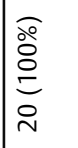 & 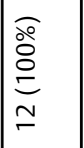 & 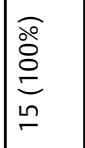 & 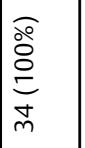 & 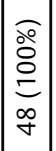 & 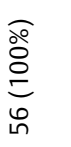 & 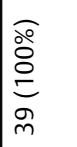 & 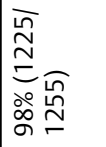 \\
\hline 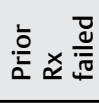 & 1 & 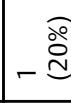 & $\simeq \stackrel{8}{\stackrel{8}{\circ}}$ & $=\frac{\partial}{\mathrm{D}}$ & बे & 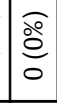 & 拿 & 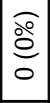 & 1 & 1 & 离 & 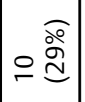 & ○े & ஓ̊ㅇㅇ & m $\stackrel{\widehat{ }}{\tilde{c}}$ & ㅇํㅁ용 \\
\hline 高高产 & \begin{tabular}{|c|} 
बळ \\
0 \\
0
\end{tabular} & ஓे & ஓें & ठ̊ํํ & 仓े & ठㅇํㅇ & \begin{tabular}{|c|} 
o̊ \\
$\stackrel{0}{0}$ \\
\end{tabular} & 仓̊ & 1 & 1 & ठ̊ํํ & ठ̊ํํ & ठ̊. & 总 & 总 & 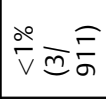 \\
\hline 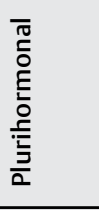 & $\stackrel{\text { ஓे }}{\stackrel{0}{0}}$ & 总 & 总 & 总 & 仓ั & ठ응 & $\mid \begin{array}{c}\text { ஓे } \\
\stackrel{8}{0} \\
0\end{array}$ & 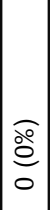 & $\frac{\stackrel{\circ}{\circ}}{\stackrel{\circ}{ }}$ & 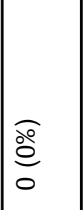 & ळ̊ํㅇ & 总 & ○े & ఏ̊ & 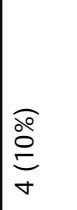 & 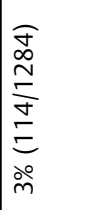 \\
\hline 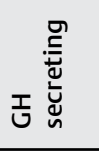 & $\begin{array}{l}\stackrel{\circ}{\stackrel{0}{0}} \\
\stackrel{\nabla}{\sigma}\end{array}$ & 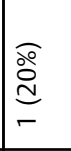 & 仓̊ํํ & $\begin{array}{l}\frac{\bar{\alpha}}{\stackrel{D}{C}} \\
m\end{array}$ & 高 & 离 & 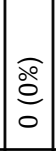 & 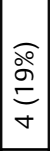 & $\begin{array}{l}\text { 方 } \\
\text { 占 } \\
\mp \\
=\end{array}$ & 总 & ஓे & 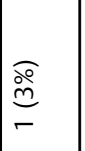 & $\begin{array}{l}\text { o. } \\
\stackrel{0}{0} \\
\circ\end{array}$ & $\begin{array}{l}\text { बơ } \\
\stackrel{0}{0} \\
\stackrel{0}{N}\end{array}$ & 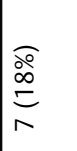 & 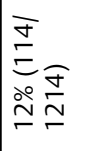 \\
\hline 謩 & 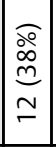 & 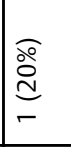 & 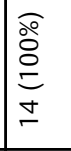 & 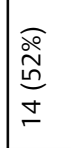 & 仓ั & 离 & 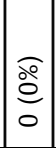 & $\begin{array}{c}\widehat{o} \\
\stackrel{m}{m} \\
\stackrel{n}{r}\end{array}$ & 离 & 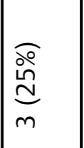 & ஓ̊ํㅇ & 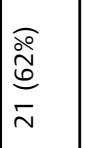 & 㐫 & 离 & 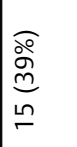 & 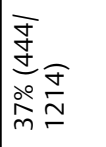 \\
\hline ¿ & $\begin{array}{l}\stackrel{0}{\circ} \\
\stackrel{2}{\circ} \\
6\end{array}$ & 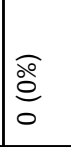 & 总 & 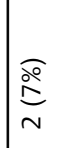 & $\begin{array}{l}\stackrel{0}{\circ} \\
\stackrel{8}{0} \\
\infty \\
\infty \\
-\end{array}$ & $\begin{array}{l}\widehat{D} \\
\stackrel{8}{0} \\
\stackrel{0}{c} \\
m \\
m\end{array}$ & 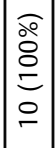 & \begin{tabular}{|c|} 
\\
$\stackrel{0}{0}$ \\
$\infty$ \\
$\stackrel{\infty}{0}$ \\
$\infty$
\end{tabular} & 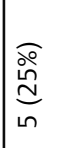 & 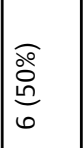 & 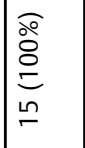 & 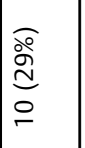 & 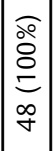 & 京 & 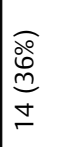 & 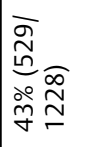 \\
\hline 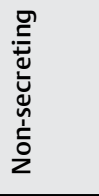 & 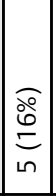 & $\begin{array}{l}\widehat{\circ} \\
\text { 。ㅇ } \\
\text { m } \\
m\end{array}$ & 总 & 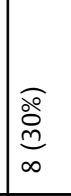 & 离 & 六 & 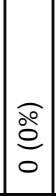 & 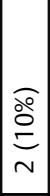 & 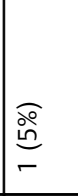 & 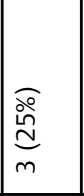 & 离 & $\frac{\widehat{O}}{\stackrel{0}{6}}$ & 离 & 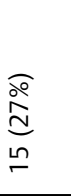 & 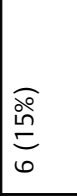 & 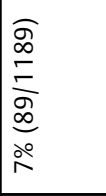 \\
\hline 娄蒙 & $\begin{array}{l}\infty \\
\vdots \\
\sigma \\
\end{array}$ & $\frac{1}{1}$ & 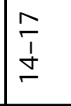 & $\stackrel{1}{1}$ & 告 & $\begin{array}{l}0 \\
I \\
1 \\
\end{array}$ & $\begin{array}{l}2 \\
\stackrel{1}{1} \\
\stackrel{1}{\simeq}\end{array}$ & $\frac{\infty}{1}$ & $\begin{array}{l}\infty \\
\frac{1}{1} \\
\llcorner\end{array}$ & $\stackrel{\sim}{m}$ & $\frac{\infty}{1}$ & $\frac{\infty}{1}$ & $\begin{array}{l}\sigma \\
\bar{\sigma} \\
\sigma\end{array}$ & 立 & $\begin{array}{l}\infty \\
\frac{1}{1} \\
\infty\end{array}$ & 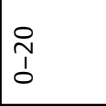 \\
\hline = & $\approx$ & in & 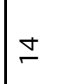 & $\grave{\sim}$ & $\stackrel{\infty}{\sim}$ & $m$ & $\circ$ & $\bar{\sim}$ & $\stackrel{N}{ }$ & $\simeq$ & $\stackrel{\llcorner}{\sim}$ & $\stackrel{*}{m}$ & $\stackrel{\infty}{+}$ & in & mे & 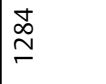 \\
\hline 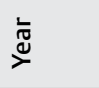 & 离 & 产 & 定 & 帝 & ڤे̀ & 訔 & $\begin{array}{l}\hat{a} \\
\grave{v}\end{array}$ & 高 & 㐫 & 을 & $\stackrel{0}{2}$ & $\bar{i}$ & $\bar{\sim}$ & $\frac{n}{2}$ & 竞 & $\stackrel{\hat{\sim}}{\stackrel{i}{2}}$ \\
\hline 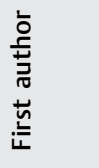 & 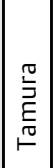 & $\frac{\stackrel{\circ}{\bar{n}}}{\frac{5}{z}}$ & $\frac{\mathscr{O}}{<}$ & 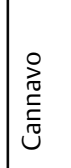 & 总 & 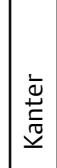 & $\begin{array}{l}\tilde{D} \\
0\end{array}$ & 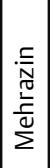 & $\frac{0}{\hat{0}}$ & 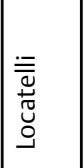 & $\mid \frac{0}{\bar{N}}$ & 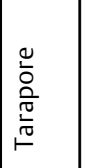 & $\begin{array}{l}\frac{r}{\frac{\pi}{n}} \\
\frac{\sqrt{n}}{n}\end{array}$ & $\frac{\sqrt{0}}{N}$ & 离 & 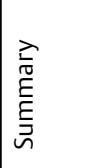 \\
\hline
\end{tabular}


Pediatric Pituitary Adenoma Perry et al. 97

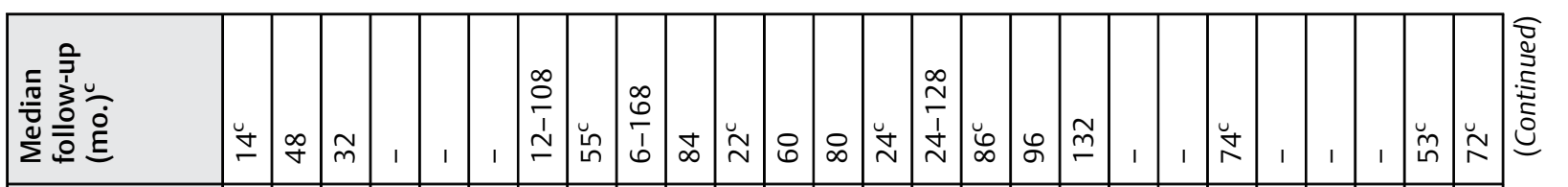

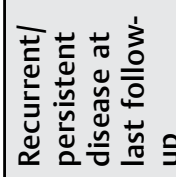

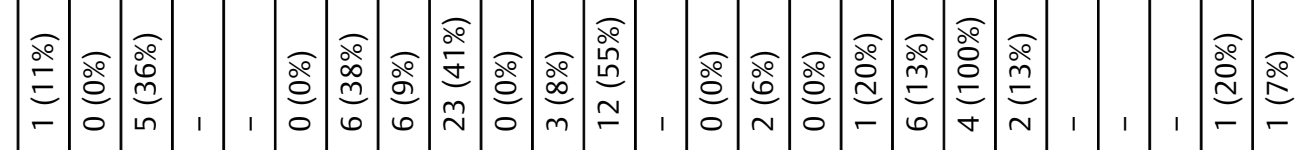

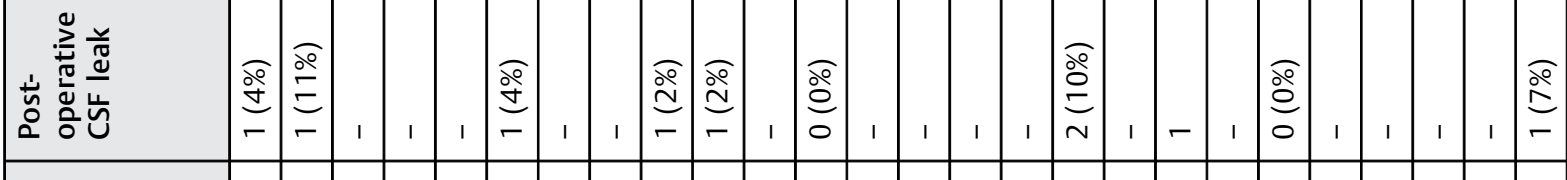

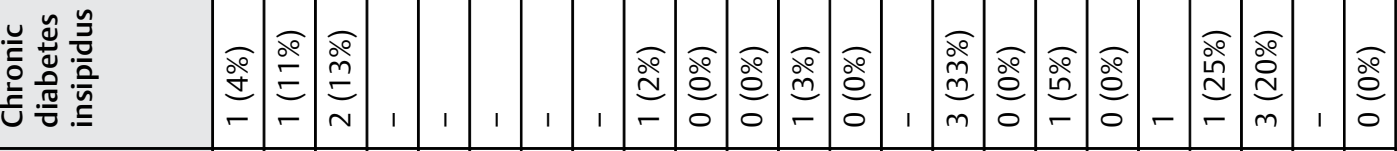

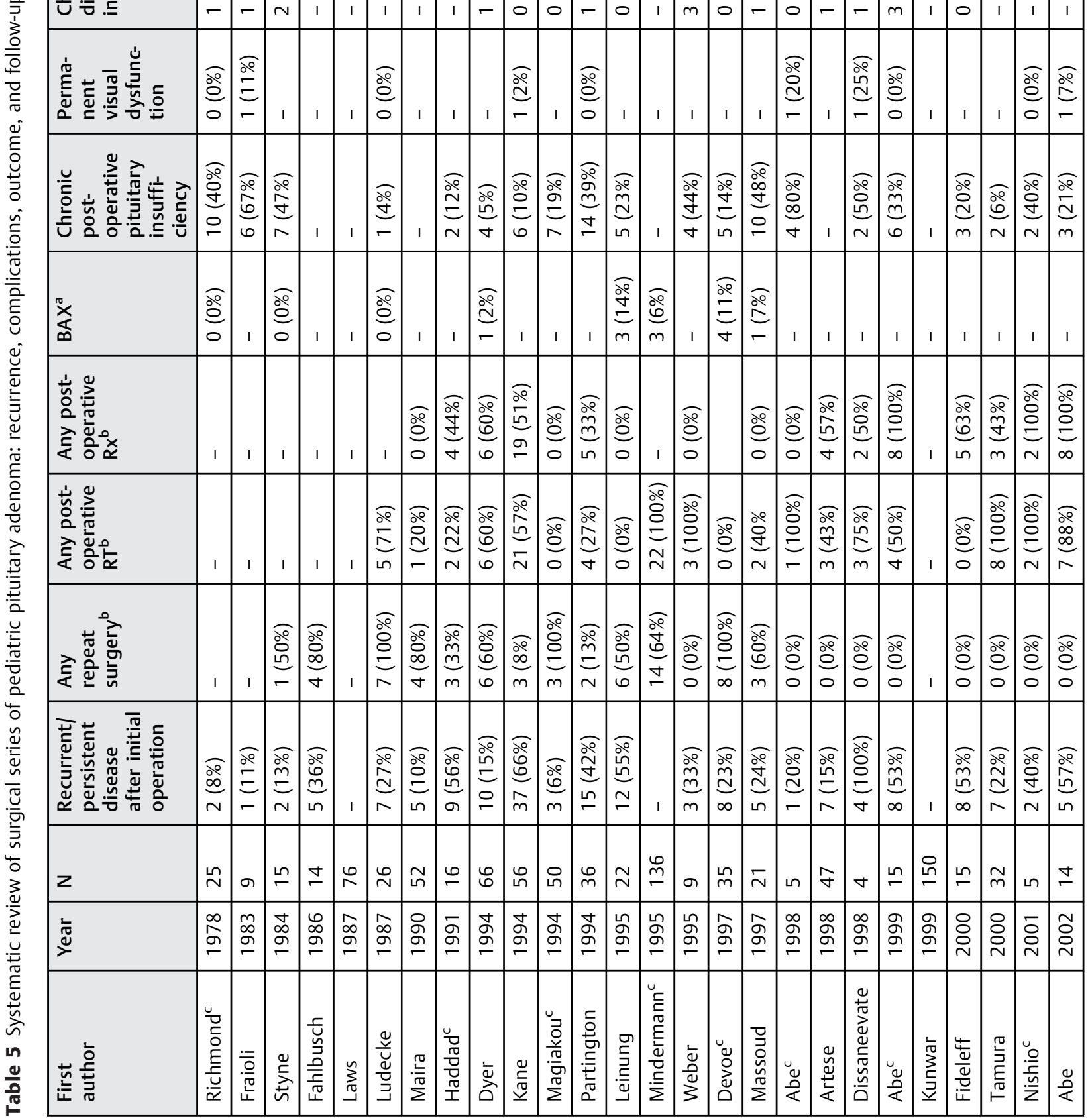




\begin{tabular}{|c|c|c|c|c|c|c|c|c|c|c|c|c|c|c|}
\hline 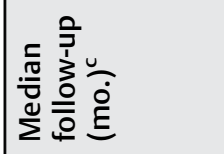 & 8 & $\underset{\infty}{\infty}$ & 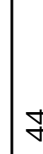 & $\infty$ & 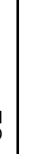 & in & & 1 & 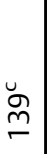 & $\stackrel{\infty}{\sim}$ & 品 & $\breve{N}$ & 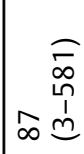 & ச্ \\
\hline 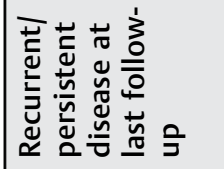 & 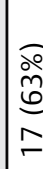 & ஓ̊ & $\frac{\text { के }}{\text { वे }}$ & ஃ & 7 & 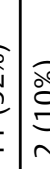 & & 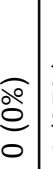 & 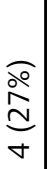 & $\frac{\widehat{\partial}}{\stackrel{\rho}{\rho}}$ & 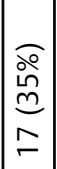 & 1 & 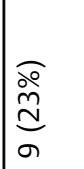 & 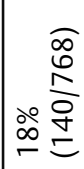 \\
\hline 竞旁 & 1 & I & वे & 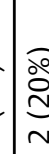 & (3) & 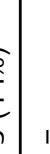 & & $\begin{array}{c}\text { ळ } \\
\stackrel{\varnothing}{\varnothing}\end{array}$ & 1 & $\mid \begin{array}{c}\widehat{o} \\
\stackrel{\circ}{0} \\
0\end{array}$ & 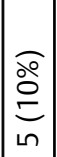 & 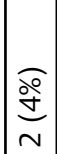 & 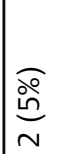 & 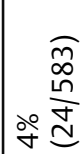 \\
\hline 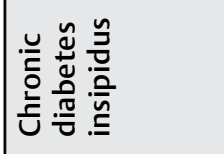 & $\begin{array}{l}\text { ㅇํㅇ } \\
\text { 굼 }\end{array}$ & 1 & $\begin{array}{l}\text { वे } \\
\text { ले } \\
\text { r }\end{array}$ & ○े & ò & । & | & $\begin{array}{c}\stackrel{\circ}{\circ} \\
\stackrel{0}{0} \\
0\end{array}$ & $\begin{array}{l}\stackrel{\curvearrowright}{\stackrel{\circ}{5}} \\
-\end{array}$ & 1 & 离 & 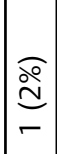 & 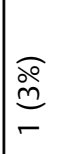 & 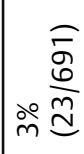 \\
\hline 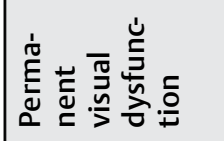 & 苘 & I & गे & । & ì & $\frac{5}{2}$ & & 1 & 1 & $\begin{array}{c}\stackrel{0}{\circ} \\
\stackrel{9}{-} \\
-\end{array}$ & 1 & 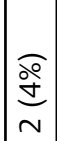 & 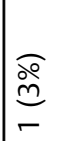 & \\
\hline 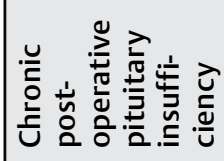 & ஓ̊̀ & I & $\begin{array}{l}\text { वे } \\
\text { वे } \\
\text { ले } \\
\text { co }\end{array}$ & I & 옹 & I & & \begin{tabular}{c|}
$\stackrel{\widehat{o}}{\infty}$ \\
- \\
-
\end{tabular} & 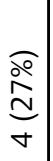 & 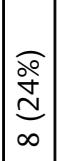 & 1 & 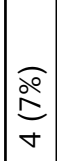 & 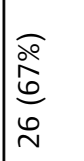 & 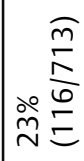 \\
\hline 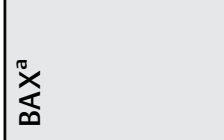 & 1 & 1 & 总 & 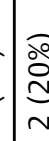 & 1. & 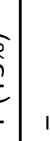 & I & 1 & 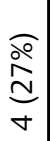 & $\mid \begin{array}{c}\stackrel{\circ}{\circ} \\
\stackrel{\varrho}{=} \\
-\end{array}$ & 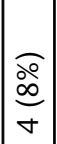 & 1 & $\widehat{\widehat{\partial}}$ & 点 \\
\hline 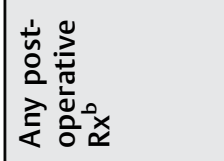 & 욤 & 응 & बे & ठㅇ & 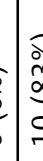 & ?ै & & $\begin{array}{c}\widehat{o} \\
\stackrel{0}{0} \\
0\end{array}$ & 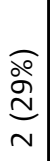 & $\mid \begin{array}{c}\circ \\
\stackrel{\circ}{0} \\
\stackrel{0}{C} \\
6\end{array}$ & 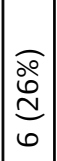 & 产 & 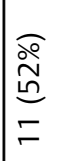 & 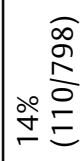 \\
\hline 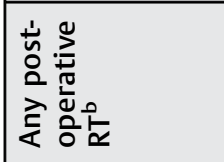 & $\underset{\infty}{\stackrel{\partial}{\mathcal{O}}}$ & 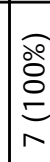 & dે & $\frac{2}{20}$ & ì & $\stackrel{0}{0}$ & 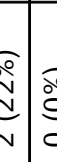 & $\begin{array}{c}\widehat{o} \\
\stackrel{0}{0} \\
0\end{array}$ & $\begin{array}{c}\stackrel{\partial}{\circ} \\
\hat{\hbar} \\
\forall \\
\forall\end{array}$ & 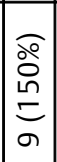 & 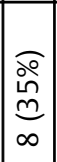 & 厌 & 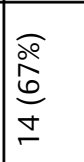 & 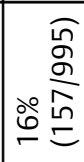 \\
\hline 空范离 & ১̊ & 仓 & $\begin{array}{l}\text { वे } \\
\stackrel{\text { n}}{m} \\
\text { m }\end{array}$ & $\sqrt{\stackrel{2}{\stackrel{9}{2}}}$ & ¿ & $\begin{array}{ll}0 \\
0 \\
0 \\
0\end{array}$ & 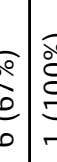 & $\begin{array}{l}0 \\
\stackrel{0}{8} \\
\varrho \\
- \\
-\end{array}$ & $\begin{array}{l}\text { ᄋ̊ } \\
\stackrel{0}{0} \\
0\end{array}$ & $\mid \begin{array}{c}\widehat{o} \\
\stackrel{\circ}{0} \\
0\end{array}$ & $\begin{array}{c}\stackrel{\bigcirc}{\stackrel{\circ}{巳}} \\
-\end{array}$ & @̊ํㅇ & 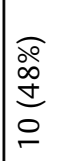 & 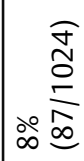 \\
\hline 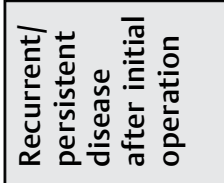 & 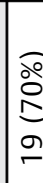 & $\begin{array}{l}\text { ळे } \\
\text { ळे } \\
\end{array}$ & בd & ठ̊ㅇ & 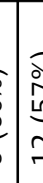 & 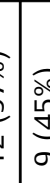 & 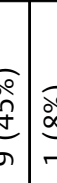 & $\begin{array}{c}\widehat{\circ} \\
\stackrel{\circ}{\infty} \\
-\end{array}$ & 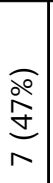 & $\mid \begin{array}{c}0 \\
\infty \\
\infty \\
\sigma \\
6\end{array}$ & 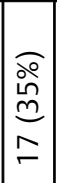 & $\mid \begin{array}{c}\overparen{0} \\
\stackrel{0}{0} \\
\stackrel{0}{0} \\
\infty \\
\sim\end{array}$ & 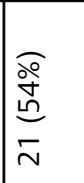 & 疍 \\
\hline$z$ & $\lesssim$ & $\stackrel{\infty}{-}$ & $\stackrel{m}{m}$ & 은 & $i$ & 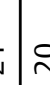 & & $\stackrel{\sim}{\sim}$ & $\stackrel{\llcorner\Omega}{\ulcorner}$ & $\stackrel{\nabla}{m}$ & $\underset{+}{\stackrel{\infty}{+}}$ & 占 & $\stackrel{m}{m}$ & 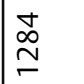 \\
\hline 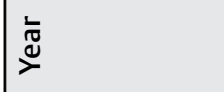 & 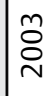 & 离 & 㞩 & 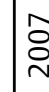 & 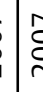 & $p_{j}^{\infty}$ & D. & $\frac{0}{2}$ & $\frac{0}{0}$ & $\bar{\Sigma}$ & $\overline{\bar{D}}$ & $\frac{\ln }{2}$ & $\hat{\tilde{N}}$ & 文 \\
\hline ڤ气 & 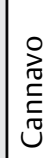 & 产 & 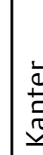 & $\tilde{\Xi}$ & 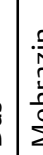 & $\sum_{3}^{2}$ & & 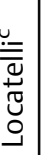 & 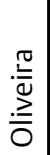 & 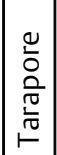 & $\mid \begin{array}{l}\frac{u}{\pi} \\
\frac{\pi}{n} \\
\frac{\pi}{n}\end{array}$ & $\begin{array}{l}u \\
\frac{\mathrm{C}}{\mathrm{N}} \\
\frac{\mathrm{N}}{\mathrm{N}}\end{array}$ & $\vec{E}$ & 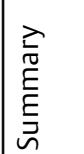 \\
\hline
\end{tabular}




\section{Primary Stereotactic Radiosurgery}

In addition to the 39 patients described above, we separately identified 2 pediatric patients with pituitary adenoma who were treated with primary SRS, rather than TSR. In the former case, an 18-year-old with prolactinoma strongly desired to minimize risk of infertility, correspondingly refused surgery, and was offered SRS as an alternative. The treatment plan consisted of 25 Gy delivered to the 50\% isodose line, to a treatment volume of $2.2 \mathrm{~cm}^{3}$ for a maximum dose of $50 \mathrm{~Gy}$. A biochemical cure was documented within 18 months, no permanent hormonal replacement therapies were required, and the patient was able to conceive as intended without fertility treatments. No recurrence has been documented in 7 years of clinical follow-up. The second patient had underlying McCune-Albright syndrome with severe fibrous dysplasia of the skull base and a radiographic adenoma that was considered GH producing by laboratory criteria, which obliterated the sphenoid sinus, precluding TSR. Correspondingly, SRS was offered, with a treatment plan of $20 \mathrm{~Gy}$ to the $60 \%$ isodose line to a total volume of $1.3 \mathrm{~cm}^{3}$, with a maximum dose of $33.3 \mathrm{~Gy}$. The patient has remained symptom free off pharmacotherapy for over 5 years of follow-up, with minimal persistent supranormal elevation of insulin-like growth factor-1 (IGF-1) and normal GH.

\section{Systematic Review}

Literature search identified 37 English language publications reporting surgical series of pediatric pituitary adenomas meeting inclusion criteria with extractable by-patient data on the outcomes of interest, spanning 1978 to 2015 (-Table 4). Together with the present series, 1,284 patients have been reported with pediatric pituitary adenoma. ACTHsecreting tumors were most frequently reported (43\%), followed by PRL-secreting (37\%), GH-secreting (12\%), and nonsecreting (7\%); plurihormonal tumors were reported in $3 \%$. Less than $1 \%$ of all tumors were radiated prior to TSR $(n=3)$, while $14 \%$ had been trialed on at least one medication. TSR was the approach of choice in $98 \%$ of patients. Extent-of-resection was only documented in $28 \%$ of cases; among those, GTR was reported in $78 \%$. Disease was cured with primary surgery in $65 \%$.

The remaining $35 \%$ were reported as having recurrent or persistent disease after the initial operation (-Table 5). Treatment paradigms were very heterogeneous, follow-up in many prior series was short, and adjuvant therapy was incompletely documented in many manuscripts; notwithstanding, among those patients with recurrent or persistent disease, at least $8 \%$ underwent repeat surgery, $16 \%$ were radiated, and $14 \%$ received postoperative pharmacotherapy. Reported complications included postoperative pituitary insufficiency requiring pharmacologic supplementation in $23 \%$, permanent visual dysfunction in $6 \%$, chronic DI in $3 \%$, and postoperative CSF leak in $4 \%$. Follow-up data was inconsistently reported, but approximate mean follow-up was 63 months (range 0-240, excluding present series). At the time of last follow-up, $18 \%$ had recurrent or persistent disease.

\section{Discussion Part One: Lessons from the Study Cohort and Literature Review}

In setting the stage for our broader survey of the topic, we reviewed our surgical series of 39 pediatric pituitary adenomas, as well as the preceding literature documenting related cohorts. Several key observations stood out, which collectively reaffirmed the disease's intrinsic challenges.

In our series and literature review, the rates of recurrent or persistent disease after primary surgery were $54 \%$ and $35 \%$, respectively, which reflect a two- to three-fold increase from large adult series that have approximated recurrence rates for nonfunctioning, PRL-secreting, ACTH-secreting, and GH-secreting tumors at $16 \%, 13 \%, 12 \%$, and $1.3 \%$, respectively. ${ }^{12,13}$ However, our finding are consistent with previous pediatric reviews, which have suggested that secretory pituitary disease is more difficult to control and prone to recurrence in children, particularly Cushing disease, which is estimated to have a $40 \% 10$-year recurrence rate in childrenalthough this conclusion has not been universally reproduced. ${ }^{2,12-16}$ Of note, the higher recurrence rate noted in the study cohort most likely reflects the observed differences in follow-up, as well as a potential underlying reporting bias, given the established tendency for studies to under-report true long-term recurrence rates-particularly in Cushing's disease. ${ }^{2,16-19}$

In the setting of tumor recurrence, adults also appear to be more easily managed than children are. In adults, repeat surgery is an effective first-line treatment for recurrent or persistent tumor without cavernous sinus involvement, and prior series have documented a biochemical cure in up to $57 \%$ of secreting tumors after a second TSR, which is a marked improvement compared with our pediatric results (30\%). ${ }^{20}$ By extension, the clinical trajectories in recurrent or persistent disease have the potential to be quite discouraging in children, with only 7 (33\%) of 21 patients reaching a cure after a single treatment for recurrence, and 8 (38\%) of 21 patients requiring treatment with at least three different modalities beyond primary TSR.

The pediatric pituitary adenoma population is also especially vulnerable to hypopituitarism, due in large part to the high incidence of recurrence and multi-modality treatments. ${ }^{21,22}$ Although most complications in the present study were rare and comparable to those associated with adult disease, permanent pituitary replacement therapy was required in $67 \%$ of our patients, as compared with 2 to $27 \%$ in major preceding adult reviews (and up to 55\% in isolated series). ${ }^{23,24}$ This contrast is in spite of the fact that hypopituitarism is strongly associated with tumor size, but pediatric tumors are more likely to be microadenomas, with a median maximum tumor diameter of $11 \mathrm{~mm}$ in the study cohort (range 1-40 mm ). ${ }^{25,26}$ Although our literature review documented a lower overall rate at $23 \%$ (range $4-80 \%$ ), this difference again most likely reflects our increased follow-up time, as well as the higher fraction of recurrent or progressive tumors in our cohort ( $54 \%$ in the study cohort, versus $35 \%$ overall), or potentially under-reporting in the literature. Regardless, the possibility that two-thirds of pediatric 
patients may suffer some degree of endocrine deficiency has dramatic implications, especially with respect to growth and development and fertility. ${ }^{2,10,27-29}$ With this in mind, we turn to an overview of the key concepts in pediatric pituitary adenoma management.

\section{Discussion, Part Two: Key Concepts in Pediatric Pituitary Adenoma Management}

\section{Epidemiology and Genetics}

Approximately 3 to $9 \%$ of pituitary adenomas occur in children, which corresponds to $3 \%$ of all pediatric intracranial neoplasms. ${ }^{2,6,7,30}$ The overall prevalence of pituitary adenoma may be increased among female children up to $2: 1$, due to the marked prolactinoma predominance in girls. $2,4,9,10,27,28,31$ Sporadic pituitary adenomas have been documented to harbor a wide range of mutations involving common tumor suppressor or oncogenes, including GNAS, PTTG, HMGA2, and FGFR-4. ${ }^{28,32,33}$ Although clear correlations between disease phenotype and underlying genetic abnormalities remain incompletely understood, several interesting relationships have been characterized-most prominently, the $40 \%$ prevalence of GNAS-activating mutations in somatotrophic tumors. ${ }^{28,34}$

Associations with genetic syndromes are rare, but potentially an important consideration in younger patients with pituitary adenoma. MEN-1 is the most common such association and has been reported to present with pituitary adenoma in children as young as 5 years. ${ }^{28,35}$ The syndrome arises in patients who inherit a single mutated allele of the menin tumor suppressor gene, and subsequently acquire a "second hit."36-39 Individuals bearing the menin mutation have a 30 to $40 \%$ lifetime risk of pituitary adenoma; 60\% of which secrete PRL and $20 \% \mathrm{GH}^{40}$

A second important association is the McCune-Albright syndrome, in which a non-heritable postzygotic activating GNAS mutation yields a range of endocrinologic derangements, café au lait spots, and polyostotic fibrous dysplasia. ${ }^{28,41,42}$ Correspondingly, pituitary surgery can be prohibitively challenging, and when undertaken, may require extensive drilling to effectively create the entire transsphenoidal working corridor. Correspondingly, SRS may be the preferred first-line treatment for these children, as in our case, described above.

Carney complex is a very rare autosomal dominant disorder characterized by endocrine hyperactivity, myxomas, lentigines, schwannomas, and adenomas, which is caused by an inactivating mutation of the PKAR1A gene in $60 \%$ of patients, though the underlying mechanism in the remaining families is incompletely understood. ${ }^{28,43,44}$ Interestingly, Carney complex patients frequently present with non-pituitary Cushing's syndrome due to primary adrenocortical neoplasms and then subsequently develop $\mathrm{GH}$-secreting pituitary adenomas, which are characteristically slow-growing and difficult to identify on imaging. ${ }^{28,44-46}$

Familial isolated pituitary adenomas (FIPA) is a term used to describe families with two or more first degree relatives developing pituitary adenomas that are negative for menin or PRKARIA mutations. ${ }^{28}$ Of the 211 families described, $\sim 20 \%$ harbor an inactivating heterozygous germline mutation of the tumor suppressor gene AIP. ${ }^{47-49}$ No definitive trends have been established regarding disease features within these patients, which may reflect the low disease penetrance. Although PRL- and GH-secreting tumors predominate, the full range of pituitary pathologies has been described.

\section{Clinical Presentation}

Pediatric pituitary adenoma presentation varies by hormonal subtype, each of which can be loosely grouped by the relative onset of symptoms. Non-secreting tumors are the least common, as they rarely have time for sufficient growth to produce symptoms while the patient remains in childhood. Correspondingly, when they do appear, these tumors generally occur in post-pubescent individuals, who are best approached and treated as young adults. ${ }^{4,8,10}$

GH-secreting tumors are uncommon, often present in prepubertal children and infants, and preferentially arise in males at a 2:1 incidence with precipitous growth, acromegaly, or headaches-although pubertal arrest or primary amenorrhea may be rare presentations of a $\mathrm{GH}$-secreting adenoma masquerading as a microprolactinoma. ${ }^{4,6} \mathrm{ACTH}$-secreting tumors occur slightly later in childhood, with peak incidence at the onset of puberty, and an overall 3:1 female predominance. ${ }^{4,6,14,50}$ Classic symptoms of hyperadrenocorticotrophism are prototypical and range from Cushingoid appearance to growth arrest, weight gain, amenorrhea, mental status changes, hypertension, and hyperglycemia. ${ }^{14,15}$

Most prior reviews and textbooks have reported PRLsecreting tumors as the most common pediatric pituitary adenomas; the vast majority of which come to clinical attention during puberty, with a 5:1 female predominance., ${ }^{4,51}$ Primary and secondary amenorrhea account for three-quarters of their presentations, while male children present with growth arrest, delayed puberty, or galactorrhea. ${ }^{4,52,53}$ Interestingly, although PRL-secreting tumors are more common overall, as our series and review demonstrate, ACTH-secreting tumors are the largest fraction of tumors that are surgically treated. ${ }^{4,27,51,54,55}$ This potentially attributable to a publication bias, particularly since there has been so much academic interest within the neurosurgical and endocrinologic communities regarding pediatric Cushing disease. ${ }^{14,15,56,57}$ More likely, this trend reflects the responsiveness of prolactinomas to pharmacotherapy and the general bias against early surgery in the pediatric population. ${ }^{28,53,58}$

In contrast to adults, children rarely present with focal neurologic signs. ${ }^{2,5,55}$ Visual dysfunction is a hallmark of the nonfunctioning macroadenomas that dominate adult disease, but occurs in fewer than $10 \%$ children-although Webb et al documented $60 \%$ in one study of 20 children, which was also notable for a higher than average incidence of macroadenoma. ${ }^{2,3,5,55}$ As Webb's cohort demonstrates, this difference can be attributed to the predominance of secreting tumors among children, who are also thought to be more physically and psychosocially sensitized to the effects of hyperprolactinemia. ${ }^{51,52,55}$ 


\section{Preoperative Endocrinologic Evaluation}

As in adults, preoperative assessment in children incorporates diagnostic and confirmatory biochemical studies, as well as focused neuroimaging, and formal neuro-ophthalmologic examination with visual field testing. Serum studies panel of anterior pituitary hormones including PRL, ACTH, $\mathrm{GH}, \mathrm{TSH}, \mathrm{LH}$, and $\mathrm{FSH}$ is requisite, both to screen for secondary subclinical abnormalities and to evaluate for possible preoperative pituitary insufficiency.

Laboratory evaluation for prolactinoma begins with a simple serum PRL assay, and although reliable reference ranges have not been definitely established in children, 5 to $25 \mathrm{ng} / \mathrm{mL}$ in girls and 5 to $15 \mathrm{ng} / \mathrm{mL}$ in boys are generally considered normal, with a peak in puberty. ${ }^{58}$ Supranormal PRL levels below $100 \mathrm{ng} / \mathrm{mL}$ may be attributable to the socalled "stalk effect," in which a macroadenoma compresses the pituitary infundibulum, decreasing tonic dopaminergic inhibition of PRL and producing the mild abnormality. ${ }^{29,59} \mathrm{~A}$ normal or mildly supranormal PRL with severe symptoms should raise suspicion for the high-dose "hook effect," especially in the setting of a large tumor. This laboratory phenomenon occurs due to an enzyme-linked immunosorbent assay (ELISA) technique that depends on two-site binding (capture and signal antibodies) for a positive result, which becomes saturated in the presence of extremely high serum PRL concentrations, "hooking" the measured value downward. $^{60-63}$ Above $100 \mathrm{ng} / \mathrm{mL}$, prolactinoma is relatively assured and certain above $200 \mathrm{ng} / \mathrm{mL}$-although results below these thresholds do not exclude the possibility of a true, secreting prolactinoma. ${ }^{53,58}$

Although serum GH concentration can be readily measured, it is subject to normal diurnal variations and is influenced by a wide swath of physiologic activities including exercise, stress, fasting states, and sleep, potentially resulting in a normal range from 0.5 to $30 \mathrm{ng} / \mathrm{mL}$ in a single day. ${ }^{64-66}$ Correspondingly, IGF-1 has been developed as a surrogate marker that reflects the overall physiologic mean $\mathrm{GH}$ value during the preceding 24 to 48 hour period. ${ }^{67}$ However, given that both $\mathrm{GH}$ and IGF-1 fluctuate with age and are physiologically elevated during adolescence and puberty, multiple measurements of both values are recommended in equivocal cases. ${ }^{6}$ In parallel, the oral glucose tolerance test (OGTT) is a highly specific confirmatory test, in which patients drink a $75 \mathrm{~g}$ glucose load; GH suppression to $<1 \mathrm{ng} / \mathrm{mL}$ within 2 hours of ingestion indicates a normal response, whereas value $>2 \mathrm{ng} / \mathrm{mL}$ is considered diagnostic, and 1 to $2 \mathrm{ng} / \mathrm{mL}$ is strongly suggestive of acromegaly. ${ }^{68}$

Cushing's disease is suggested by hypercortisolism with elevated serum ACTH levels: concentrations from 5 to $20 \mathrm{pg} / \mathrm{mL}$ are highly consistent with an ACTH-dependent process and $>20 \mathrm{pg} / \mathrm{mL}$ are diagnostic. ${ }^{69-71}$ Ectopic ACTH production must subsequently be ruled out, typically using a combination of high-dose dexamethasone suppression (HDDS) and corticotropin-releasing hormone (CRH) stimulation tests. ${ }^{72}$ In true Cushing's disease, overnight administration of oral high-dose dexamethasone will reduce 8 am cortisol to $<5 \mathrm{mcg} / \mathrm{dL}$ (or below $50 \%$ of baseline), while intravenous injection of $\mathrm{CRH}$ results in a marked increase in both ACTH and cortisol within 45 minutes-an effect that can be potentiated by pre-treating with vasopressin, although that is rarely required in children (positive test thresholds are specific to the center and protocol). ${ }^{73,74}$ Ectopic ACTH generally does not respond to either agent. Positive results on both HDDS and CRH is highly specific for Cushing's disease, and a positive CRH test coupled with an unambiguous adenoma on pituitary magnetic resonance imaging (MRI) is considered diagnostic; however, absent positive imaging and conflicting results between HDDS and $\mathrm{CRH}$ may prompt inferior petrosal sinus sampling (IPSS) for diagnosis and lateralization.

In this test, the bilateral inferior petrosal dural venous sinuses are endovascularly canalized and sampled. ACTH values from centrally drawn samples are then compared with ones from the peripheral blood, and an ACTH gradient $>2$ is diagnostic of Cushing's disease, with a $95 \%$ sensitivity and $93 \%$ specificity. ${ }^{75}$ These results can be further elevated to 95 to $100 \%$ sensitivity and specificity by administering CRH and using a diagnostic threshold $>3 .{ }^{76}$ However, minor proximal misplacement of the catheter may yield a false negative, and although the rate of serious complications is quite low, rare cerebrovascular accidents or cranial nerve palsies have been reported, and the logistics of completing the procedure in children are potentially complex. ${ }^{77-79}$ Correspondingly, our practice has been to avoid subjecting children to this intervention whenever possible (it was required in 1 of 14 ACTHsecreting tumors in the study cohort). Finally, although IPSS is a potentially powerful diagnostic tool in patients with equivocal biochemistry, its use as a lateralization technique to guide hemi-hypophysectomy is more controversial. Several studies have reported successful localization resulting in biochemical cure in 71 to $74 \%$ of patients; however, others have failed to reproduce this result or improve significantly on the baseline odds of $50 \%{ }^{14,76,80,81}$

\section{Imaging and Ophthalmologic Assessment}

Contrast-enhanced MRI with thin (1-3 mm) coronal slices through the sella is the bedrock of pituitary adenoma imaging and provides essential information for diagnosis and surgical planning (-Fig. 2). Arbitrarily, pituitary adenomas have been traditionally separated into micro- and macroadenomas using the $10-\mathrm{mm}$ maximum diameter threshold, although both are commonly seen in children, microadenomas predominate, given the predominance of secreting lesions. ${ }^{10,82,83}$ Prolactinomas are the exception to this principle and have a more expansive growth pattern that predisposes to macroadenoma formation-particularly in young males-as well as a tendency to present in older children who are more likely to harbor larger tumors. ${ }^{28}$

On routine sequences, adenomas are frequently appreciable on pre-contrast T1-weighted images as well demarcated hypointense regions when compared with normal gland-a differentiation that is augmented by the normal gland's robust gadolinium-uptake on contrast-enhanced scans. Contrast-enhanced images are particularly important in the assessment of ACTH-secreting microadenomas, which are typically the smallest lesions and the most likely to 


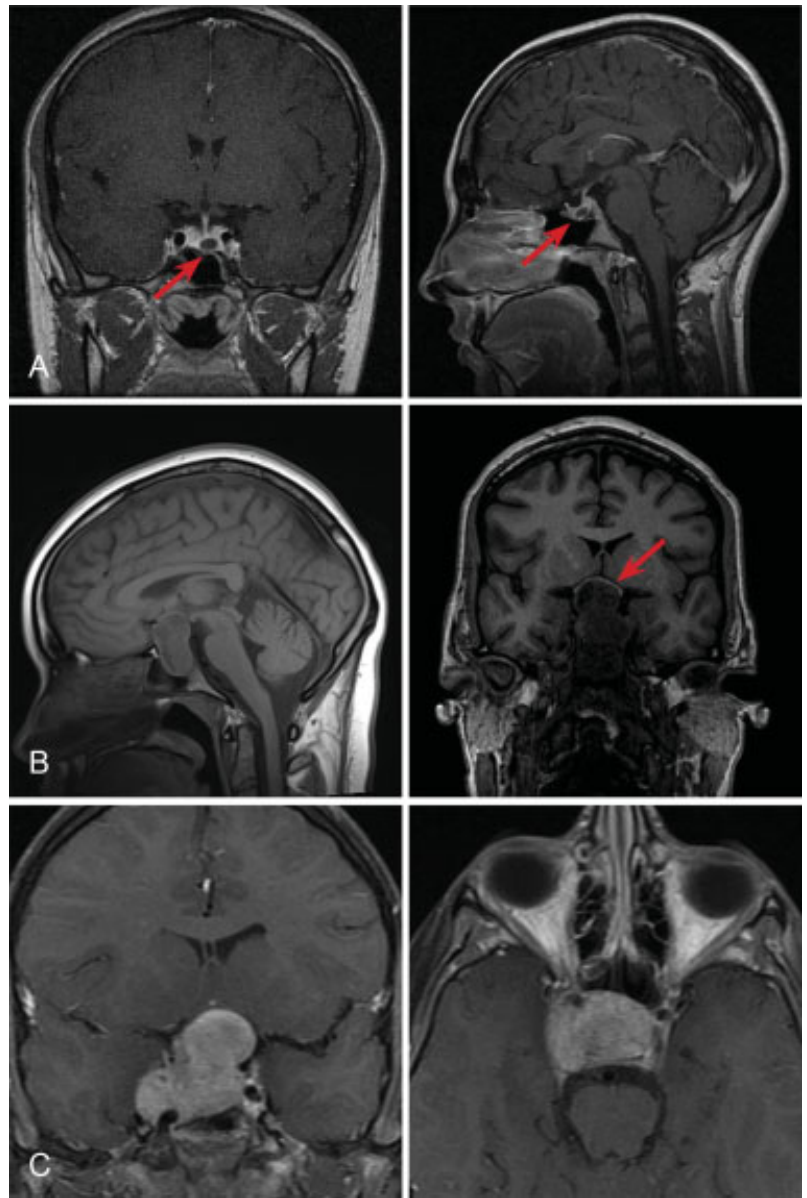

Fig. 2 Gadolinium-enhanced T1-weighted MRI of the brain in the coronal and sagittal planes ( $\mathrm{A}$ and $\mathbf{B}$ ) demonstrates a hypo-enhancing eccentric left sellar masses (red arrows) surrounded by briskly enhancing normal hypophyseal tissue, characteristic of pituitary microadenoma. Pre-contrast sagittal T1-weighted and coronal MPRAGE images (B) demonstrate a large, well-circumscribed, sellar mass with surrounding benign bony remodeling, significant superior displacement of the optic chiasm (red arrow), and internal heterogeneity, consistent with a partially hemorrhagic pituitary macroadenoma. Gadolinium-enhanced T1-weighted coronal and axial images (C) demonstrate a large, vividly enhancing sellar mass, with invasion of the bilateral cavernous sinuses, encasement of the internal carotid arteries, and significant suprasellar and middle fossa extension, suggestive of an aggressive pituitary macroadenoma. MPRAGE, magnetization prepared rapid acquisition gradient echo; MRI, magnetic resonance imaging.

enhance. ${ }^{83,84}$ Dynamic MR techniques rely on rapidly repeated scans, which capture the wash-in and wash-out of contrast to demonstrate a time-dependent pattern of early gland enhancement, followed by delayed adenoma enhancement, optimizing visualization of the lesion.

While microadenomas may be difficult to identify, macroadenomas are self-evident lesions that fill and frequently expand the sella or invade the cavernous sinuses and are much more likely to demonstrate internal heterogeneity due to hemorrhage or necrosis-especially if bromocriptine therapy was previously attempted ${ }^{83,85}$ Although not universally necessary, non-contrast head computed tomography (CT) is an important adjunct in atypical lesions where craniophar- yngioma or meningioma is on the differential. In these circumstances, calcification or hyperostosis favors an alternative diagnosis, while a purely intrasellar lesion with benign bony expansion is more likely to indicate adenoma. ${ }^{86}$

Although visual dysfunction is uncommon in children due to the low incidence of macroadenoma, ophthalmologic evaluation with visual field testing is recommended where possible. The purpose is two-fold: first, awareness and articulation of subtle visual symptoms is less reliable in children; and second, it provides formal documentation of the patient's preoperative baseline.

\section{Medical Management and The Role of Deferred Surgery}

Although TSR is the preferred first-line treatment for most pituitary tumors, prolactinomas warrant a trial of medical management with dopamine agonists before a surgical intervention is considered. Cabergoline is typically more effective and better tolerated than its pharmacologic predecessor bromocriptine, with stable biochemical remission documented in $70 \%$ of macroadenomas and 80 to $90 \%$ of microadenomas. ${ }^{87-90}$ Cabergoline also has the advantages of a once- or twice-weekly 0.25 to $2 \mathrm{mg}$ dose formulation and decreased incidence of major adverse events including hemorrhage and spontaneous CSF leak-although intolerable side effects remain the chief etiology of treatment failure. $^{91-93}$ Of note, female patients desiring fertility should be preferentially placed on bromocriptine, as it had a more well-characterized safety profile. ${ }^{94-98}$

In some individuals, medical monotherapy may provide a sustained cure. ${ }^{99}$ Colao et al reported 64 to $69 \%$ sustained remission at 5 years after a 2-year treatment period with cabergoline, a marked improvement over 7 to $38 \%$ described previously after cessation of bromocriptine. ${ }^{99-106}$ Still other new data on pergolide, lisuride, and quinagolide have demonstrated comparable or superior efficacy to cabergoline with respect to biochemical remission and tumor regression; however, each is still awaiting the Food and Drug Administration approval, particularly with respect to the potential risk of valve disease in association with chronic exposure to these agents. ${ }^{29,107-109}$ These findings are promising; however, given the elevated risk of recurrence in younger patients, extrapolations to the pediatric population are guarded.

While the majority of patients with prolactinoma will benefit from an initial trial of medical management, particularly in the pediatric population, there are several relative indications for early surgical intervention, including acute visual loss or cranial nerve palsy. ${ }^{29,110,111}$ As these sequelae typically occur in large, invasive macroadenomas, a surgical cure may not be obtained, but decompression relieves mass effect, and tumor cytoreduction will potentiate response to anti-dopaminergic therapy. ${ }^{112,113}$ Multi-modal therapy is often required in these patients, in particular SRS, to treat cavernous sinus disease, but TSR is almost always preferred route for acute decompression of the optic apparatus. ${ }^{29}$ Similarly, patients who have a very low probability of tumor control with pharmacotherapy may benefit from 
initial surgical treatment, as dopamine agonists may increase tumor fibrosis, predisposing to a more challenging resection. $^{114}$

By contrast, the somatostatin analog octreotide has been shown to biochemically normalize GH hypersecretion in up to $55 \%$ of adults and induce a degree of radiographic tumor remission in 25 to $70 \%$, but has not been shown to provide a durable disease cure, and the potential risks of life-long therapy in children are not established. ${ }^{115-117}$ Some prior studies have demonstrated improved surgical cure rates after octreotide pretreatment; however, this has not been consistently reproduced, and neither a dose-response relationship nor an ideal duration-of-pretreatment is established. ${ }^{118-120}$ Correspondingly, we do not recommend the first-line medical therapy for most children with GH-secreting pituitary adenomas. ${ }^{117}$

\section{Transsphenoidal Surgery, Skull Base Techniques, and Special Consideration in Pediatrics}

TSR is the preferred treatment for pituitary adenoma in the overwhelming majority of circumstances, particularly given that most are limited to the sellar or midline suprasellar regions. ${ }^{5,11,121,122}$ Sellar microadenomas predominate in the pediatric population, making a large fraction potentially amendable to primary TSR; however, sphenoid sinus pneumatization has the potential to limit the operative corridor. Although first observed as early as 6 months in some children, the pneumatization process predominantly occurs during years 3 to 7 , and the completion may take until the child is 9 to $12 .^{123-125}$

In some patients with partial pneumatization, the midline sphenoid bone can potentially be removed with a high-speed drill to provide access to the sella, which is often preferable to a transcranial approach for small, intrasellar lesions. ${ }^{8,56,126}$ Radiology-based anatomic studies have described approximate drilling distances by age group, which can be correlated with preoperative imaging (ideally, a stereotactic CT scan). ${ }^{127,128}$ of note, even among the youngest children studied, clival inter-carotid distances never prohibited transsphenoidal surgery. However, pedicled nasoseptal flaps are difficult to raise in patients aged $<10$ years and questionable in patients 10 to 13 years, potentially limiting reconstructive options if an elevated risk of CSF leak is anticipated. ${ }^{129}$ Finally, even modern endoscopic instruments may still be very large for safe, efficient use in smaller nares; correspondingly, a sublabial approach may be preferred in up to $39 \%{ }^{130}$ Additionally, image guidance may be extremely helpful to guide the drilling necessary to better establish a transsphenoidal corridor.

A related technical question is centered on the comparison between microscopic and endoscopic techniques for pediatric pituitary tumor resection. In the adult population, this question has been interrogated for pituitary adenoma as well as a wide range of other midline cranial base neoplasms, with generally equivocal findings. Results have varied widely between centers and surgeons, and EEA is generally accepted as a non-inferior alternative to microsurgery. Most reports suggest EEA has improved rates of GTR and improvement of visual function and decreased rates of pituitary insufficiency but there has been concern of a higher rate of carotid artery injury. ${ }^{131-138}$ Few prospective trials comparing EEA and microsurgery have been completed, with five meeting criteria for inclusion in a recent meta-analysis. ${ }^{139-145}$ Although the overall evidence level and data quality were quite low, the study concluded that EEA is associated with significantly lower complication rates, but not biochemical cure, as compared with microscopic TSR. Further prospective study is clearly required to answer this question more definitively, particularly in children.

Neither prospective studies have compared the techniques in children, nor has any retrospective study specifically taken up the EEA question in pediatric pituitary adenoma. Massimi et al reviewed a 31-patient series comparing 14 sublabial microsurgical and 17 EEA operations in a mixed population of pediatric neoplasms that included adenomas, but with a majority of craniopharyngiomas. ${ }^{146}$ Mean ages were comparable at 11.4 and 10.2 years, and there were no significant differences between the groups preoperatively. Tumor control and complication rates were not significantly different, although EEA was associated with fewer pediatric intensive care unit (PICU) admission, shorter hospitalizations, and lower pain scores. Rigante et al reported another mixed series comparing 11 sublabial microsurgical and 10 EEA operations from the same group, with comparable results. ${ }^{147}$ In addition to these direct comparisons, several other authors have published self-referencing series juxtaposing newer endoscopic results to prior microsurgical series, most of which have concluded that extent-of-resection, pituitary insufficiency, and CSF leak are stable, but not significantly improved after EEA. ${ }^{126,136,148,149}$

Advocates of EEA in the pediatric population suggest that it is associated with decreased trauma to the anterior nasopharynx (no nasal speculum) and a faster, less morbid recovery. ${ }^{150}$ Opponents highlight longer operative times, the theoretically increased risk of carotid injury, and the need for a wider corridor, potentially mandating more extensive drilling of incompletely pneumatized sinuses. EEA was previously thought to risk disruption of the craniofacial growth plates, predisposing to deformity; although rational, this hypothesis has been disproven, with no cases of delayed disfigurement identified in the several large series publishing the first long-term perspectives on EEA in children. $48,126,148,151$

A final consideration regarding EEA for pediatric pituitary adenoma is the finding that, in individual surgeons and the neurosurgical community at large, adoption of EEA has a clear learning curve, with significantly worse outcomes anticipated during the earliest phase. ${ }^{152-156}$ Given the scarcity of pediatric tumors requiring TSR, the significant morbidity associated with a poor surgical outcome, and the relative youth of the approach-particularly as compared with the depth of experience among more senior practitioners of transsphenoidal microsurgery-we recommend that treatment for pediatric pituitary tumors be concentrated in centers-of-excellence and eschew the use of EEA by inexperienced surgeons. 
Although uncommon in children, significant suprasellar tumor extension beyond the midline corridor and into the Sylvian fissure presents an important indication for transcranial or combined approaches. ${ }^{157,158}$ In many of these tumors, pituitary function is already severely compromised; therefore, the endocrine risks of accessing the sella laterally are less pronounced. However, a prefixed chiasm may present a daunting obstacle; therefore, in such cases, a pterional approach is typically preferred, as it allows the shortest and most direct possible transcranial trajectory to the sub- and retrochiasmatic spaces. ${ }^{159}$ By contrast, in patients with a postfixed chiasm and significant tumor between the optic nerves or extending anterior to the tuberculum sella, a subfrontal or transbasal approach may warrant consideration-including the unilateral subfrontal, which minimizes risk to the frontal sinus or olfactory system.

For especially large, expansive tumors and recurrences that extend along the sellar and parasellar axes, anterolateral approaches can be expanded via orbitozygomatic or orbitaloptic osteotomies, allowing greater access with minimized frontal lobe retraction. ${ }^{159,160}$ Less frequently indicated are transpetrosal or transcavernous approaches; however, they may prove useful in cases of large, invasive pituitary adenoma with significant extension throughout the retrochiasmatic, interpeduncular, or prepontine spaces. Rarely, remarkably aggressive tumors are reported with widespread posterior fossa involvement, and these lateral skull base techniques are requisite for debulking. ${ }^{161,162}$

\section{Focused Review of Pathologic Features}

The pathologic classification of pituitary tumors is extensive, and based on a combination of features including hormonal content, cell type, and ultrastructural morphology, which collectively outline 18 specific adenoma subtypes as of the 2004 World Health Organization (WHO) guidelines. ${ }^{163-166}$ Each adenoma subtype has predictable biologic patterns of behavior, with implications in terms of capacity for recurrence, overall prognosis, and response to treatment. Although these patterns have been derived from adult populations, the dominant pathologies are in parallel among children, with granulated PRL cell adenoma, densely granulated growth hormone cell adenoma, and densely granulated corticotroph adenoma comprising $27.0 \%, 7.1 \%$, and $9.6 \%$, respectively of all pituitary tumors, and therefore the overwhelming majority of secreting adenomas. ${ }^{163,165,166}$ Characteristic corticotroph-type tumors show diffuse adenoma cells, with loss of typical reticulated nesting, and diffuse ACTH positive staining (- Fig. 3A-C).

Two interesting pathologic subtypes were observed at high rates among our patients: Crooke's cell adenoma and atypical adenoma. In ACTH-secreting tumors, pathologic accumulation of perinuclear cytokeratin within the suppressed normal gland cells is a common and clinically insignificant feature termed Crooke's hyaline change. However, when these changes are observed within adenoma cells, the diagnosis of a Crooke's cell adenoma is made, which is an aggressive but benign variant carrying a $60 \%$ risk of recurrence and $24 \%$ chance of multiple recurrence. ${ }^{167}$ Character- istic pathologic features include faint perinuclear ACTH staining with correspondingly strong CAM5.2 staining ( - Fig. 3D-F). Among the four patients diagnosed with Crooke's cell adenoma in our cohort, two were cured at primary TSR, one had two recurrences requiring repeat TSR followed by PBRT before a biochemical cure was established, and the final patient remained severely symptomatic in spite of multi-modality treatment including EBRT, multiple repeat TSRs, and a craniotomy, highlighting the potential for these tumors to be remarkably aggressive, particularly in recurrence-prone pediatric patients.

Atypical pituitary adenoma is defined by the presence of mitoses, $\mathrm{K}_{\mathrm{i}}-67$ index $>3 \%$, and nuclear p53 staining with nuclear pleomorphism (-Fig. 3G). ${ }^{168,169}$ Adult series have approximated 3 to $15 \%$ incidence, as compared with the very rare $0.2 \%$ prevalence of pituitary carcinoma, with no clear correlation established between specific atypical features and disease phenotype. ${ }^{165,166,170,171}$ In our series, we encountered five atypical adenomas (13\%): all had complex histories requiring multi-modality treatment, and only one was ultimately cured. Taken together with the lack of reliable pathologic predictors of clinical behavior, we recommend close follow-up of all atypical lesions and prompt, aggressive treatment of any recurrence.

\section{Management of Progression or Recurrence}

Encouragingly, a significant fraction of pediatric pituitary adenomas do quite well following initial resection, with our own series and the literature review documenting a surgical cure in $46 \%$ and $65 \%$, respectively. Notwithstanding, recurrent or persistent disease is a common, potentially morbid, and frequently often multiply occurring management challenge in pediatric patients.

The best choice for second-line therapy is very dependent on the characteristics of the recurrence and the patient. In patients with an anatomically accessible lesion, repeat surgery is typically offered, particularly if there was a period of apparent disease remission following the initial resection. Successful treatment with a second operation was observed in $14 \%$ of our patients and up to $57 \%$ in prior series of secreting tumors in adults. ${ }^{20,172}$ However, many patients fail repeat surgery, and a large fraction have recurrent or progressive disease due to cavernous sinus involvement, which requires consideration of alternative modalities.

Pharmacotherapy is frequently trialed if repeat surgery is failed or not offered; however, patients with prolactinoma and many with GH-secreting lesions will have failed preoperative medical therapy and are unlikely to achieve durable symptomatic or biochemical disease control. Additionally, as recurrence indicates a more aggressive disease phenotype, treatment with the goal of a definitive cure is recommended. A specific exception is made for pre- or peripubertal children without severe symptoms, in whom temporizing with medication to delay radiation may be recommended-particularly if they are cabergoline- or octreotide-naïve. Combination therapies may also be effective, for example the addition of cabergoline or the $\mathrm{GH}$ receptor agonist pegvisomant to octreotide, which has been shown to be act synergistically in controlling recurrent 



Fig. 3 Histopathologic photomicrographs demonstrating a corticotroph-type tumor with typical features including diffuse adenoma cells (A, H\&E, 200X), loss of typical reticulated nesting (B, Reticulin, 200X), and diffusely positive immunohistochemical staining for ACTH (C, ACTH, 200X). Crooke's cell adenoma, with characteristic strongly positive perinuclear CAM5.2 staining (D and E, CAM5.2, 400X), and a corresponding haloing of perinuclear ACTH positivity (F, ACTH, 400X). Atypical pituitary adenoma, demonstrating two mitoses (arrowheads) in a high-powered field (G, H\&E, 400X). ACTH, adrenocorticotropic hormone; H\&E, hematoxylin and eosin.

GH-secreting adenomas in adults. ${ }^{116,117}$ Of note, all anti-tumor medications should be discontinued prior to radiation if at all possible, as dopamine and somatostatin antagonist appear to confer a radio-protective effect on tumor cells. ${ }^{173-175}$

In adults, non-operative pituitary recurrences respond quite favorably to radiation-in particular, SRS. Prior series have reported treatment success in $97 \%$ of nonsecreting tumors and 45 to $93 \%$ secreting adenomas, with Pollock estimating an overall success rate of durable biochemical cure in at least $60 \%$ of recurrent secreting tumors. ${ }^{174-180}$ Hypopituitarism is the most common complication, with 10 to $12 \%$ of adults requiring chronic hormonal supplementation after SRS. ${ }^{16,48,181,182}$

Data on pediatric pituitary radiotherapy is more limited, due to its infrequent use; as our literature review demonstrates, radiation of any modality was reported in only $16 \%$ of children with recurrent or persistent disease. This reflects a general attitude of reluctance given the pronounced risk of hypopituitarism, as well as the more general (but still rare) complications of radiation in a young population with benign disease. GH deficiency in particular has been reported in up to 86 to $100 \%$ of pediatric patients after radiation, with rare reports describing symptomatic post-radiation deficiencies in the full range of anterior pituitary hormones. ${ }^{16,183}$ Although this can be managed with supplementation, most patients still do not reach mid-parental target height. ${ }^{57,184,185}$ Complications notwithstanding, our own results and those studies that have specifically reported outcomes in pediatric secretory disease have demonstrated compelling efficacy, with local control rates of 64 to $100 \%$ after recurrence across all modalities and tumor subtypes. ${ }^{14,16,130}$

No study has yet compared EBRT and SRS in pediatric pituitary adenoma. Thoren et al reported a landmark series on SRS as primary treatment for pediatric Cushing's disease in 1986; eight patients were treated, of whom seven were cured, while one went on to BAX for persistent disease, and all eight required chronic pituitary supplementation. ${ }^{183}$ In our series, 5 (36\%) of 14 recurrences treated with radiation failed; however, when stratified by modality, 7 (70\%) of 10 SRS and $1(100 \%)$ PBRT patient were ultimately cured, as 
compared with 1 (25\%) of 4 EBRT treatments. Based on the available data and our clinical experience, we recommend SRS over EBRT in patients with symptomatic recurrences refractory to medical treatment whose tumors have $3-\mathrm{mm}$ margin between the optic nerve and the lesion, and a treatment volume $<3 \mathrm{~cm}^{3}$. This disposition is further augmented by extrapolations from the adult population and data on pediatric radiation in malignant disease, which suggest a significantly increased long-term risk of cognitive impairment or the development of a radiation-induced neoplasm following EBRT, as well as faster remission of endocrine symptoms after SRS. ${ }^{16,130,179,182,186-188}$

Large tumors abutting the optic nerve may still be managed using SRS and careful dose planning keeping the maximum optic nerve point dose $<10$ to $12 \mathrm{~Gy}$; however, this may reduce the chance for biochemical cure in a hormoneproducing tumor, as these usually require at least $20 \mathrm{~Gy}$ marginal doses. Alternatively, some centers recommend fractionated SRS, IMRT, or EBRT, supported by varying degrees of evidence. ${ }^{48,181,182}$ Overall experience with PBRT for pediatric pituitary adenoma remains quite limited at present; however, preliminary adult series have reported post-radiation hypopituitarism in as few as $30 \%$ of patients with comparable local control to SRS, suggesting that it may become an important alternative modality as access expands and costs decline. ${ }^{182,186,189,190}$ With respect to the broader clinical picture, patients undergoing radiation are recommended to discontinue any pituitary-suppressive pharmacotherapies for 2 to 4 weeks, to promote tumor cell division and therefore radiosensitivity.

Although ACTH-secreting adenomas are often radiosensitive, severe Cushing's disease has the potential to be both disabling and treatment resistant. BAX provides durable correction of symptomatic hypercortisolemia and was previously considered a preferable alternative to radiation in children. However, the treatment requires lifelong hormonal supplementation, and the decrease in negative feedback on adenoma cells resulting from the BAX may lead to a rapid and dangerous adenoma growth known as Nelson-Salassa syndrome, which is thought to be more prevalent and aggressive among younger patients. ${ }^{191}$ Correspondingly, radiation is recommended prior to BAX in most pediatric cases, reserving BAX for those cases that fail both repeat surgery and radiation. If $\mathrm{BAX}$ is required for rapid correction of severe hypercortisolemia, prophylactic SRS may be offered concurrently, which has been shown to significantly decrease the risk of Nelson-Salassa syndrome in adults. ${ }^{192}$ However, given our previous finding that a subset of Nelson-Salassa patients experience an indolent natural history, waiting for tumor growth following BAX is our preferred approach, in radiation-naïve children. ${ }^{191,192}$

Rarely, atypical pituitary adenomas, carcinomas, or instances of Nelson-Salassa syndrome may be refractory to multi-modality treatments, as in two of our patients. Trials of chemotherapeutic agents in pituitary disease have been disappointing, but nevertheless they represent a potential last line of defense. ${ }^{170}$ Temozolomide, a well-tolerated deoxyribonucleic acid (DNA)-alkylating agent that is widely used in glioma treatment, has demonstrated better efficacy than preceding chemotherapeutic regimens, with an overall clinical or radiographic response rate of 60 to $69 \%{ }^{170,193-197}$ Newer targeted therapies are also undergoing active investigation as second-line, concomitant, or alternative agents in aggressive pituitary adenoma, including the anti-vascular endothelial growth factor (anti-VEGF) monoclonal antibody bevacizumab, mammalian target of rapamycin (mTOR) inhibitor everolimus, and epidermal growth factor receptor (EGFR)2 inhibitor lapatinib. ${ }^{198-201}$ At present, data are very limited even in the adult population, and the risk-benefit calculus of trialing any chemotherapy in a child will be determined on an individualized basis-although by this point in the natural history, patients have usually aged beyond the elevated risks of pediatric care.

\section{Major Complications and their Management}

Although a broad range of complications has been documented after pituitary adenoma treatment, most are rare occurrences, with pituitary insufficiency, DI, and CSF leak comprising the majority of significant treatment consequence. As described above, symptomatic deficiencies of anterior pituitary hormones are the most frequent complications of both surgery and radiation, with chronic pharmacologic supplementation required in $\sim 25 \%$ after surgery, $10 \%$ after radiation, and up to two-thirds complex patients with extended follow-up, as in the study own cohort. GH deficiency is the most common, with significant implications in children with respect to overall growth potential, as well as onset and duration of puberty. Thyroid and corticotropin deficiencies occur less frequently, but management with supplementation is uncomplicated and rarely morbid; gonadotropin deficiency is rare in the absence of panhypopituitarism, but may require treatment for secondary infertility. ${ }^{6,202}$ In women with prolactinomas who retain normal gonadotropin function, inducing biochemical remission using bromocriptine is generally sufficient to promote normal fertilization; however, conception and obstetric care for women with refractory disease is potentially complex and may require an experienced reproductive endocrinologist. ${ }^{203,204}$

Though typically transient, DI nevertheless has the potential to be a major management challenge and potentially life threatening in its most serious iterations. Macroadenomas, invasive or aggressive lesions, and patients presenting with subclinical sodium derangements at baseline are at especially high risk, but in all patients an elevated index of suspicion is warranted if postoperative urine output is brisk. ${ }^{145,148,205}$ Pediatric resuscitation goals vary by age and weight, but core treatment principles include early administration of oral or subcutaneous desmopressin acetate (DDAVP), urine replacement with half-normal saline, and serial serum sodium checks. ${ }^{206}$ Most patients recover in hours-to-days; however, some undergo poly-phasic cycles of polyuria and antidiuresis, while $\sim 3 \%$ develop stable euvolemic disease requiring chronic DDAVP. $^{54}$

Postoperative CSF leak has been estimated in 3 to $8 \%$ of pediatric TSR cases and been reported in up to $20 \%$ in some individual series, with significant risk factors including tumors 


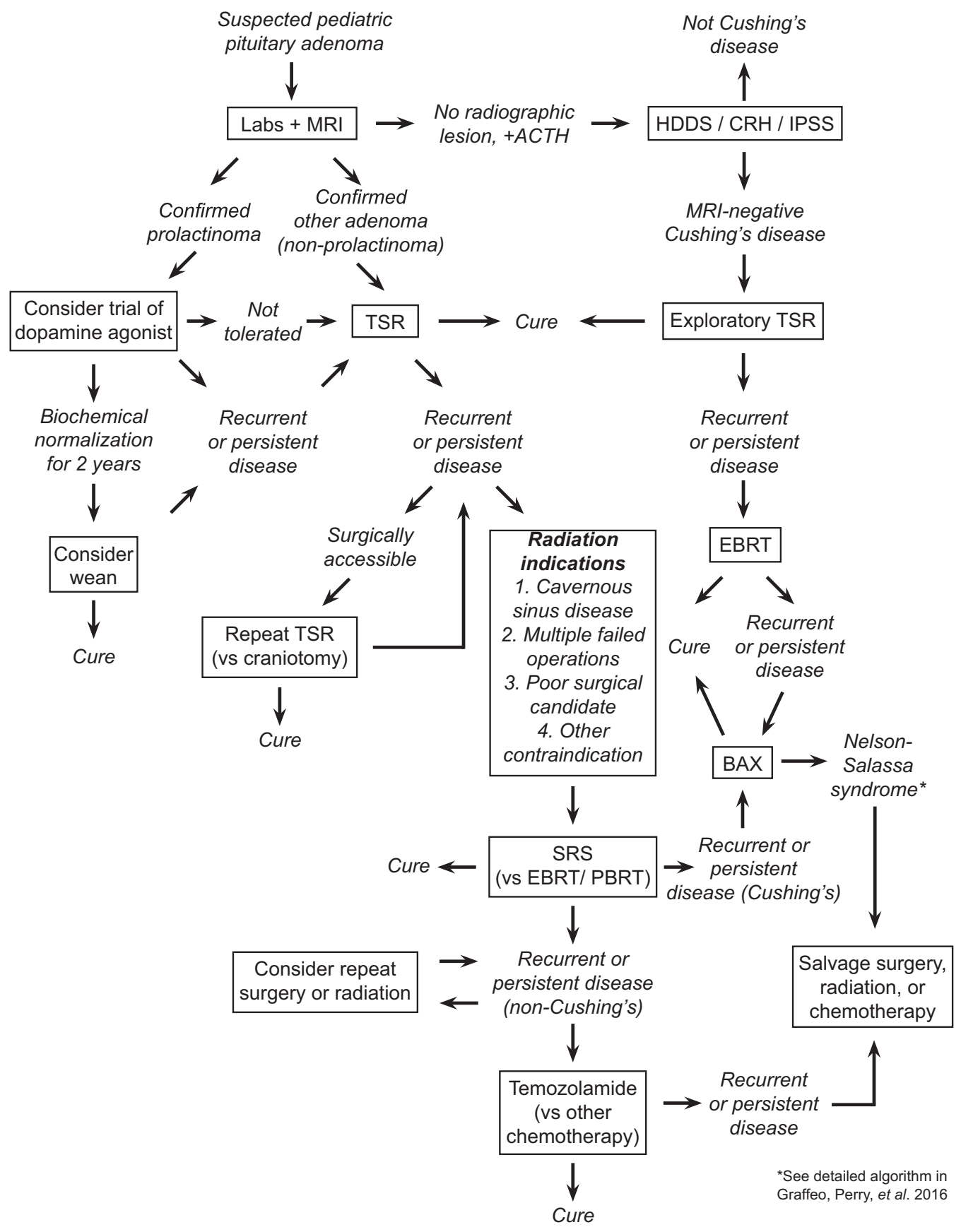

Fig. 4 Treatment algorithm for pediatric pituitary adenoma. ACTH, adrenocorticotropic hormone; BAX, bilateral adrenalectomy; CRH, corticotropin releasing hormone; EBRT, external beam radiotherapy; HDDS, high-dose dexamethasone suppression; IPSS, inferior petrosal sinus sampling; MRI, magnetic resonance imaging; PBRT, proton beam radiotherapy; SRS, stereotactic radiosurgery; TSR, transsphenoidal resection.

with suprasellar extension, intraoperative CSF leak, or prior treatment with surgery, radiation, or dopamine agonists. $^{92,136,207-209}$ General management strategies are comparable to adults, with the specific exception that children younger than 10 to 13 years may not have adequate tissue to support a vascularized nasoseptal flap. ${ }^{129}$ Similarly, although lumbar drainage may be attempted as a first-line intervention -potentially in combination with acetazolamide-the procedure may require sedation in children, and the drain itself is more prone to inadvertent removal. Correspondingly, most leaks are better managed via exploration and repair. Simple defects may be adequately treated with abdominal fat graft; however, larger fistulas or leaks in patients who have been radiated or multiply operated are more likely to be successfully treated with a nasoseptal flap or a comparable autograft, and multi-layer repair is universally recommended. ${ }^{208-211}$

\section{Conclusion}

Pediatric pituitary adenomas are a diverse and remarkably challenging family of tumors; the ideal management of which is subject to a broad range of potentially complicating factors including restrictive anatomy, the predominance of secretory disease, and the potentially heightened 
vulnerability of these children to both treatment and disease morbidity. Complicating matters further, this vulnerability to major, life-altering endocrine dysfunction, such as infertility or growth arrest, may exert its own confounding influence on treatment patterns and disease natural history. By way of example, many studies have concluded that children are at higher risk of adenoma recurrence, yet it remains unknown whether this is attributable to a true phenotypic difference in disease aggressiveness or a reflection of a subtly more conservative treatment paradigm and almost impossible to discern retrospectively.

Notwithstanding, based on the available data, we have observed that most patients respond well to surgery and experience a swift and uncomplicated recovery; however, recurrent or persistent disease appears to be more frequent in children than in adults and may be more difficult to manage and marked by serial recurrences requiring multimodality therapy. Ultimately, the plan of care must be tailored to the individual patient and tumor; however, we have consolidated our overarching strategy, and standard practices are consolidated into a treatment algorithm that can be adapted to the demands of specific cases ( $\mathbf{- F i g . 4}$ ).

In general terms, prolactinomas are trialed on cabergoline, while other adenomas and prolactinomas failing medical therapy or presenting with significant neurologic symptoms are offered surgery. Recurrent or persistent tumors are offered repeat surgery where anatomically feasible. Those recurrences not amenable to surgery may be successfully temporized with medications-particularly in prepubescent patients with mild symptoms-but the majority of these patients will ultimately require radiation, typically via single-fraction SRS. Cases of severe Cushing's disease may ultimately necessitate BAX, while extremely aggressive adenomas and carcinomas are potentially candidates for chemotherapy, with the caveat that these highly complex cases will inevitably require the most tailored and potentially unconventional treatment plans. Taken together, the study cohort and literature review inform our perspective on this challenging entity, but perhaps most importantly, they highlight the need for better evidence, and the development of an adaptive framework for translating the study of a rare and highly variable disease into rational, individualized patient care.

\section{Previous Presentations}

Components of this work were presented or submitted as abstracts at the NASBS 2016 and CNS 2017.

\section{Conflicts of interest}

None.

\section{Acknowledgment}

The authors would like to thank Dr. Aditya Raghunathan for his expert input and provision of histopathologic photomicrographs.

\section{References}

1 Espay AJ, Azzarelli B, Williams LS, Bodensteiner JB. Recurrence in pituitary adenomas in childhood and adolescence. J Child Neurol 2001;16(05):364-367
2 Kane LA, Leinung MC, Scheithauer BW, et al. Pituitary adenomas in childhood and adolescence. J Clin Endocrinol Metab 1994;79 (04):1135-1140

3 Maira G, Anile C. Pituitary adenomas in childhood and adolescence. Can J Neurol Sci 1990;17(01):83-87

4 Mindermann T, Wilson CB. Pediatric pituitary adenomas. Neurosurgery 1995;36(02):259-268, discussion 269

5 Partington MD, Davis DH, Laws ER Jr, Scheithauer BW. Pituitary adenomas in childhood and adolescence. Results of transsphenoidal surgery. J Neurosurg 1994;80(02):209-216

6 Abe T, Tara LA, Lüdecke DK. Growth hormone-secreting pituitary adenomas in childhood and adolescence: features and results of transnasal surgery. Neurosurgery 1999;45(01):1-10

7 Artese R, D'Osvaldo DH, Molocznik I, et al. Pituitary tumors in adolescent patients. Neurol Res 1998;20(05):415-417

8 Dyer EH, Civit T, Visot A, Delalande O, Derome P. Transsphenoidal surgery for pituitary adenomas in children. Neurosurgery 1994; 34(02):207-212, discussion 212

9 Kunwar S, Wilson CB. Pediatric pituitary adenomas. J Clin Endocrinol Metab 1999;84(12):4385-4389

10 Lafferty AR, Chrousos GP. Pituitary tumors in children and adolescents. J Clin Endocrinol Metab 1999;84(12):4317-4323

11 Massoud AF, Powell M, Williams RA, Hindmarsh PC, Brook CG. Transsphenoidal surgery for pituitary tumours. Arch Dis Child 1997;76(05):398-404

12 Jane J, Thapar K, Laws E. Pituitary tumors: functioning and nonfunctioning. Youmans Neurological Surgery. Philadelphia, PA: Elsevier Saunders; 2011:1476-1510

13 Jane JA Jr, Laws ER Jr. The surgical management of pituitary adenomas in a series of 3,093 patients. J Am Coll Surg 2001;193 (06):651-659

14 Joshi SM, Hewitt RJ, Storr HL, et al. Cushing's disease in children and adolescents: 20 years of experience in a single neurosurgical center. Neurosurgery 2005;57(02):281-285, discussion 281285

15 Shah NS, George J, Acharya SV, et al. Cushing disease in children and adolescents: twenty years' experience in a tertiary care center in India. Endocr Pract 2011;17(03):369-376

16 Storr HL, Plowman PN, Carroll PV, et al. Clinical and endocrine responses to pituitary radiotherapy in pediatric Cushing's disease: an effective second-line treatment. J Clin Endocrinol Metab 2003;88(01):34-37

17 Atkinson AB, Kennedy A, Wiggam MI, McCance DR, Sheridan B. Long-term remission rates after pituitary surgery for Cushing's disease: the need for long-term surveillance. Clin Endocrinol (Oxf) 2005;63(05):549-559

18 Bochicchio D, Losa M, Buchfelder M. Factors influencing the immediate and late outcome of Cushing's disease treated by transsphenoidal surgery: a retrospective study by the European Cushing's Disease Survey Group. J Clin Endocrinol Metab 1995; 80(11):3114-3120

19 Rees DA, Hanna FW, Davies JS, Mills RG, Vafidis J, Scanlon MF. Long-term follow-up results of transsphenoidal surgery for Cushing's disease in a single centre using strict criteria for remission. Clin Endocrinol (Oxf) 2002;56(04):541-551

20 Benveniste RJ, King WA, Walsh J, Lee JS, Delman BN, Post KD. Repeated transsphenoidal surgery to treat recurrent or residual pituitary adenoma. J Neurosurg 2005;102(06):1004-1012

21 Friedman RB, Oldfield EH, Nieman LK, et al. Repeat transsphenoidal surgery for Cushing's disease. J Neurosurg 1989;71(04): 520-527

22 Patil CG, Veeravagu A, Prevedello DM, Katznelson L, Vance ML, Laws ER Jr. Outcomes after repeat transsphenoidal surgery for recurrent Cushing's disease. Neurosurgery 2008;63(02):266-270 , discussion 270-271

23 Baskin DS, Boggan JE, Wilson CB. Transsphenoidal microsurgical removal of growth hormone-secreting pituitary adenomas. A review of 137 cases. J Neurosurg 1982;56(05):634-641 
24 Sudhakar N, Ray A, Vafidis JA. Complications after trans-sphenoidal surgery: our experience and a review of the literature. $\mathrm{Br} \mathrm{J}$ Neurosurg 2004;18(05):507-512

25 Fatemi N, Dusick JR, Mattozo C, et al. Pituitary hormonal loss and recovery after transsphenoidal adenoma removal. Neurosurgery 2008;63(04):709-718, discussion 718-719

26 Nomikos P, Ladar C, Fahlbusch R, Buchfelder M. Impact of primary surgery on pituitary function in patients with nonfunctioning pituitary adenomas - a study on 721 patients. Acta Neurochir (Wien) 2004;146(01):27-35

27 Katavetin P, Cheunsuchon P, Swearingen B, Hedley-Whyte ET, Misra M, Levitsky LL. Review: Pituitary adenomas in children and adolescents. J Pediatr Endocrinol Metab 2010;23(05): 427-431

28 Keil MF, Stratakis CA. Pituitary tumors in childhood: update of diagnosis, treatment and molecular genetics. Expert Rev Neurother 2008;8(04):563-574

29 Liu JK, Couldwell WT. Contemporary management of prolactinomas. Neurosurg Focus 2004;16(04):E2

30 Faglia G, Spada A. Genesis of pituitary adenomas: state of the art. J Neurooncol 2001;54(02):95-110

31 Nishio S, Morioka T, Suzuki S, Takeshita I, Fukui M, Iwaki T. Pituitary tumours in adolescence: clinical behaviour and neuroimaging features of seven cases. J Clin Neurosci 2001;8(03): 231-234

32 Alexander JM, Biller BM, Bikkal H, Zervas NT, Arnold A, Klibanski A. Clinically nonfunctioning pituitary tumors are monoclonal in origin. J Clin Invest 1990;86(01):336-340

33 Spada A, Mantovani G, Lania A. Pathogenesis of prolactinomas. Pituitary 2005;8(01):7-15

34 Heaney AP, Melmed S. Molecular targets in pituitary tumours. Nat Rev Cancer 2004;4(04):285-295

35 Stratakis CA, Schussheim DH, Freedman SM, et al. Pituitary macroadenoma in a 5-year-old: an early expression of multiple endocrine neoplasia type 1. J Clin Endocrinol Metab 2000;85 (12):4776-4780

36 Marx SJ, Agarwal SK, Kester MB, et al. Multiple endocrine neoplasia type 1: clinical and genetic features of the hereditary endocrine neoplasias. Recent Prog Horm Res 1999;54:397-438, discussion 438-439

37 Spada A. Genetic aspects of pituitary tumors. J Pediatr Endocrinol Metab 2001;14(Suppl 5):1213-1216, discussion 1261-1262

38 Thakker RV, Bouloux P, Wooding C, et al. Association of parathyroid tumors in multiple endocrine neoplasia type 1 with loss of alleles on chromosome 11. N Engl J Med 1989;321(04): 218-224

39 Vergès B, Boureille $F$, Goudet $P$, et al. Pituitary disease in MEN type 1 (MEN1): data from the France-Belgium MEN1 multicenter study. J Clin Endocrinol Metab 2002;87(02):457-465

40 Asa SL, Ezzat S. The pathogenesis of pituitary tumours. Nat Rev Cancer 2002;2(11):836-849

41 Akintoye So, Chebli C, Booher S, et al. Characterization of gspmediated growth hormone excess in the context of McCune-Albright syndrome. J Clin Endocrinol Metab 2002;87(11):5104-5112

42 Koch G, Tiwisina T. [Contribution to the heredity of acromegaly and hyperostosis generalisata with pachyderma (chromophobe hypophysis adenoma in father and son]. Arztl Forsch 1959; 13:489-504

43 Boikos SA, Stratakis CA. Carney complex: pathology and molecular genetics. Neuroendocrinology 2006;83(3-4):189-199

44 Boikos SA, Stratakis CA. Pituitary pathology in patients with Carney Complex: growth-hormone producing hyperplasia or tumors and their association with other abnormalities. Pituitary 2006;9(03):203-209

45 Kurtkaya-Yapicier O, Scheithauer BW, Carney JA, et al. Pituitary adenoma in Carney complex: an immunohistochemical, ultrastructural, and immunoelectron microscopic study. Ultrastruct Pathol 2002;26(06):345-353
46 Pack SD, Kirschner LS, Pak E, Zhuang Z, Carney JA, Stratakis CA. Genetic and histologic studies of somatomammotropic pituitary tumors in patients with the "complex of spotty skin pigmentation, myxomas, endocrine overactivity and schwannomas" (Carney complex). J Clin Endocrinol Metab 2000;85(10):3860-3865

47 Beckers A, Aaltonen LA, Daly AF, Karhu A. Familial isolated pituitary adenomas (FIPA) and the pituitary adenoma predisposition due to mutations in the aryl hydrocarbon receptor interacting protein (AIP) gene. Endocr Rev 2013;34(02): 239-277

48 Guaraldi F, Storr HL, Ghizzoni L, Ghigo E, Savage MO. Paediatric pituitary adenomas: a decade of change. Horm Res Paediatr 2014;81(03):145-155

49 Tichomirowa MA, Barlier A, Daly AF, et al. High prevalence of AIP gene mutations following focused screening in young patients with sporadic pituitary macroadenomas. Eur J Endocrinol 2011; 165(04):509-515

50 Fraioli B, Ferrante L, Celli P. Pituitary adenomas with onset during puberty. Features and treatment. J Neurosurg 1983;59 (04):590-595

51 Cannavò S, Venturino M, Curtò L, et al. Clinical presentation and outcome of pituitary adenomas in teenagers. Clin Endocrinol (Oxf) 2003;58(04):519-527

52 Dissaneevate P, Warne GL. Hyperprolactinaemia and pituitary adenomas in adolescence. J Pediatr Endocrinol Metab 1998;11 (04):531-541

53 Fideleff HL, Boquete HR, Sequera A, Suárez M, Sobrado P, Giaccio A. Peripubertal prolactinomas: clinical presentation and longterm outcome with different therapeutic approaches. J Pediatr Endocrinol Metab 2000;13(03):261-267

54 Albright AL, Pollack IF, Andelson PD. Principles and Practice of Pediatric Neurosurgery. New York, NY:Thieme;2015

55 Webb C, Prayson RA. Pediatric pituitary adenomas. Arch Pathol Lab Med 2008;132(01):77-80

56 Oliveira RS, Castro Md, Antonini SR, Martinelli CE Jr, Moreira AC, Machado HR. Surgical management of pediatric Cushing's disease: an analysis of 15 consecutive cases at a specialized neurosurgical center. Arq Bras Endocrinol Metabol 2010;54 (01):17-23

57 Styne DM, Grumbach MM, Kaplan SL, Wilson CB, Conte FA. Treatment of Cushing's disease in childhood and adolescence by transsphenoidal microadenomectomy. N Engl J Med 1984; 310(14):889-893

58 Abe T, Lüdecke DK. Transnasal surgery for prolactin-secreting pituitary adenomas in childhood and adolescence. Surg Neurol 2002;57(06):369-378, discussion 378-379

59 Arafah BM, Nekl KE, Gold RS, Selman WR. Dynamics of prolactin secretion in patients with hypopituitarism and pituitary macroadenomas. J Clin Endocrinol Metab 1995;80(12):3507-3512

60 Barkan AL, Chandler WF. Giant pituitary prolactinoma with falsely low serum prolactin: the pitfall of the "high-dose hook effect": case report. Neurosurgery 1998;42(04):913-915, discussion 915-916

61 Comtois R, Robert F, Hardy J. Immunoradiometric assays may miss high prolactin levels. Ann Intern Med 1993;119(02):173

62 Fleseriu M, Lee M, Pineyro MM, et al. Giant invasive pituitary prolactinoma with falsely low serum prolactin: the significance of 'hook effect'. J Neurooncol 2006;79(01):41-43

63 Frieze TW, Mong DP, Koops MK. "Hook effect" in prolactinomas: case report and review of literature. Endocr Pract 2002;8(04): 296-303

64 Chapman IM, Hartman ML, Straume M, Johnson ML, Veldhuis JD, Thorner MO. Enhanced sensitivity growth hormone (GH) chemiluminescence assay reveals lower postglucose nadir GH concentrations in men than women. J Clin Endocrinol Metab 1994; 78(06):1312-1319

65 Iranmanesh A, Grisso B, Veldhuis JD. Low basal and persistent pulsatile growth hormone secretion are revealed in normal and 
hyposomatotropic men studied with a new ultrasensitive chemiluminescence assay. J Clin Endocrinol Metab 1994;78(03): 526-535

66 Ribeiro-Oliveira AJr, Barkan A. The changing face of acromegalyadvances in diagnosis and treatment. Nat Rev Endocrinol 2012;8 (10):605-611

67 Stoffel-Wagner B, Springer W, Bidlingmaier F, Klingmüller D. A comparison of different methods for diagnosing acromegaly. Clin Endocrinol (Oxf) 1997;46(05):531-537

68 Carmichael JD, Bonert VS, Mirocha JM, Melmed S. The utility of oral glucose tolerance testing for diagnosis and assessment of treatment outcomes in 166 patients with acromegaly. J Clin Endocrinol Metab 2009;94(02):523-527

69 Katznelson L, Bogan JS, Trob JR, et al. Biochemical assessment of Cushing's disease in patients with corticotroph macroadenomas. J Clin Endocrinol Metab 1998;83(05):1619-1623

70 Newell-Price J, Trainer P, Besser M, Grossman A. The diagnosis and differential diagnosis of Cushing's syndrome and pseudoCushing's states. Endocr Rev 1998;19(05):647-672

71 Woo YS, Isidori AM, Wat WZ, et al. Clinical and biochemical characteristics of adrenocorticotropin-secreting macroadenomas. J Clin Endocrinol Metab 2005;90(08):4963-4969

72 Grossman AB, Howlett TA, Perry L, et al. CRF in the differential diagnosis of Cushing's syndrome: a comparison with the dexamethasone suppression test. Clin Endocrinol (Oxf) 1988;29 (02):167-178

73 Dickstein G, DeBold CR, Gaitan D, et al. Plasma corticotropin and cortisol responses to ovine corticotropin-releasing hormone (CRH), arginine vasopressin (AVP), CRH plus AVP, and CRH plus metyrapone in patients with Cushing's disease. J Clin Endocrinol Metab 1996;81(08):2934-2941

74 Orth DN, DeBold CR, DeCherney GS, et al. Pituitary microadenomas causing Cushing's disease respond to corticotropin-releasing factor. J Clin Endocrinol Metab 1982;55(05):1017-1019

75 Kaltsas GA, Giannulis MG, Newell-Price JD, et al. A critical analysis of the value of simultaneous inferior petrosal sinus sampling in Cushing's disease and the occult ectopic adrenocorticotropin syndrome. J Clin Endocrinol Metab 1999;84(02): 487-492

76 Oldfield EH, Doppman JL, Nieman LK, et al. Petrosal sinus sampling with and without corticotropin-releasing hormone for the differential diagnosis of Cushing's syndrome. N Engl J Med 1991;325(13):897-905

77 Gandhi CD, Meyer SA, Patel AB, Johnson DM, Post KD. Neurologic complications of inferior petrosal sinus sampling. AJNR Am J Neuroradiol 2008;29(04):760-765

78 Lefournier V, Gatta B, Martinie M, et al. One transient neurological complication (sixth nerve palsy) in 166 consecutive inferior petrosal sinus samplings for the etiological diagnosis of Cushing's syndrome. J Clin Endocrinol Metab 1999;84(09):3401-3402

79 Miller DL, Doppman JL, Peterman SB, Nieman LK, Oldfield EH, Chang R. Neurologic complications of petrosal sinus sampling. Radiology 1992;185(01):143-147

80 López J, Barceló B, Lucas T, et al. Petrosal sinus sampling for diagnosis of Cushing's disease: evidence of false negative results. Clin Endocrinol (Oxf) 1996;45(02):147-156

81 Tabarin A, Greselle JF, San-Galli F, et al. Usefulness of the corticotropin-releasing hormone test during bilateral inferior petrosal sinus sampling for the diagnosis of Cushing's disease. J Clin Endocrinol Metab 1991;73(01):53-59

82 Tien RD, Kucharczyk J, Bessette J, Middleton M. MR imaging of the pituitary gland in infants and children: changes in size, shape, and MR signal with growth and development. AJR Am J Roentgenol 1992;158(05):1151-1154

83 Yousem DM, Grossman RI. Neuroradiology: The Requisites. 3rd ed. Philadelphia, PA: Mosby/Elsevier; 2010

84 Batista D, Courkoutsakis NA, Oldfield EH, et al. Detection of adrenocorticotropin-secreting pituitary adenomas by magnetic resonance imaging in children and adolescents with cushing disease. J Clin Endocrinol Metab 2005;90(09):5134-5140

85 Yousem DM, Arrington JA, Zinreich SJ, Kumar AJ, Bryan RN. Pituitary adenomas: possible role of bromocriptine in intratumoral hemorrhage. Radiology 1989;170(1 Pt 1):239-243

86 Zimmerman RA. Imaging of intrasellar, suprasellar, and parasellar tumors. Semin Roentgenol 1990;25(02):174-197

87 Casanueva FF, Molitch ME, Schlechte JA, et al. Guidelines of the Pituitary Society for the diagnosis and management of prolactinomas. Clin Endocrinol (Oxf) 2006;65(02):265-273

88 dos Santos Nunes V, El Dib R, Boguszewski CL, Nogueira CR. Cabergoline versus bromocriptine in the treatment of hyperprolactinemia: a systematic review of randomized controlled trials and meta-analysis. Pituitary 2011;14(03):259-265

89 Molitch ME, Elton RL, Blackwell RE, et al. Bromocriptine as primary therapy for prolactin-secreting macroadenomas: results of a prospective multicenter study. J Clin Endocrinol Metab 1985;60(04):698-705

90 Webster J, Piscitelli G, Polli A, Ferrari CI, Ismail I, Scanlon MF; Cabergoline Comparative Study Group. A comparison of cabergoline and bromocriptine in the treatment of hyperprolactinemic amenorrhea. N Engl J Med 1994;331(14):904-909

91 De Bellis A, Colao A, Savoia A, et al. Effect of long-term cabergoline therapy on the immunological pattern and pituitary function of patients with idiopathic hyperprolactinaemia positive for antipituitary antibodies. Clin Endocrinol (Oxf) 2008;69(02): 285-291

92 Perry A, Graffeo CS, Copeland WR III, et al. Delayed Cerebrospinal Fluid rhinorrhea after gamma knife radiosurgery with or without preceding transsphenoidal resection for pituitary pathology. World Neurosurg 2017;100:201-207

93 Schlechte JA, Sherman BM, Chapler FK, VanGilder J. Long term follow-up of women with surgically treated prolactinsecreting pituitary tumors. J Clin Endocrinol Metab 1986;62 (06):1296-1301

94 Molitch ME. Management of prolactinomas during pregnancy. J Reprod Med 1999;44(12, Suppl)1121-1126

95 Molitch ME. Pregnancy and the hyperprolactinemic woman. N Engl J Med 1985;312(21):1364-1370

96 Schlechte JA. Clinical practice. Prolactinoma. N Engl J Med 2003; 349(21):2035-2041

97 Skrabanek P, McDonald D, Meagher D, et al. Clinical course and outcome of thirty-five pregnancies in infertile hyperprolactinemic women. Fertil Steril 1980;33(04):391-395

98 Weiss MH. Medical and surgical management of functional pituitary tumors. Clin Neurosurg 1981;28:374-383

99 Colao A, Di Sarno A, Cappabianca P, Di Somma C, Pivonello R, Lombardi G. Withdrawal of long-term cabergoline therapy for tumoral and nontumoral hyperprolactinemia. N Engl J Med 2003;349(21):2023-2033

100 Johnston DG, Hall K, Kendall-Taylor P, Patrick D, Watson M, Cook DB. Effect of dopamine agonist withdrawal after long-term therapy in prolactinomas. Studies with high-definition computerised tomography. Lancet 1984;2(8396):187-192

101 Liuzzi A, Dallabonzana D, Oppizzi G, et al. Low doses of dopamine agonists in the long-term treatment of macroprolactinomas. N Engl J Med 1985;313(11):656-659

102 van 't Verlaat JW, Croughs RJ. Withdrawal of bromocriptine after long-term therapy for macroprolactinomas; effect on plasma prolactin and tumour size. Clin Endocrinol (Oxf) 1991;34(03): $175-178$

103 Wang C, Lam KS, Ma JT, Chan T, Liu MY, Yeung RT. Long-term treatment of hyperprolactinaemia with bromocriptine: effect of drug withdrawal. Clin Endocrinol (Oxf) 1987;27(03):363-371

104 Warfield A, Finkel DM, Schatz NJ, Savino PJ, Snyder PJ. Bromocriptine treatment of prolactin-secreting pituitary adenomas may restore pituitary function. Ann Intern Med 1984;101(06): 783-785 
105 Wu ZR, Zhang Y, Cai L, et al. Long-term clinical outcomes of invasive giant prolactinomas after a mean ten-year followup. Int J Endocrinol 2016;2016:8580750

106 Zárate A, Canales ES, Cano C, Pilonieta CJ. Follow-up of patients with prolactinomas after discontinuation of long-term therapy with bromocriptine. Acta Endocrinol (Copenh) 1983;104(02): 139-142

107 Orrego JJ, Chandler WF, Barkan AL. Pergolide as primary therapy for macroprolactinomas. Pituitary 2000;3(04):251-256

108 Webster J. Cabergoline and quinagolide therapy for prolactinomas. Clin Endocrinol (Oxf) 2000;53(05):549-550

109 Webster J. A comparative review of the tolerability profiles of dopamine agonists in the treatment of hyperprolactinaemia and inhibition of lactation. Drug Saf 1996;14(04):228-238

110 Amar AP, Couldwell WT, Chen JC, Weiss MH. Predictive value of serum prolactin levels measured immediately after transsphenoidal surgery. J Neurosurg 2002;97(02):307-314

111 Tyrrell JB, Lamborn KR, Hannegan LT, Applebury CB, Wilson CB. Transsphenoidal microsurgical therapy of prolactinomas: initial outcomes and long-term results. Neurosurgery 1999;44(02): 254-261, discussion 261-263

112 Thorner MO. Prolactinoma. West J Med 1983;139(05):703-705

113 Vance ML, Thorner MO. Prolactinomas. Endocrinol Metab Clin North Am 1987;16(03):731-753

114 Bevan JS, Webster J, Burke CW, Scanlon MF. Dopamine agonists and pituitary tumor shrinkage. Endocr Rev 1992;13(02):220-240

115 Abe T, Lüdecke DK. Effects of preoperative octreotide treatment on different subtypes of $90 \mathrm{GH}$-secreting pituitary adenomas and outcome in one surgical centre. Eur J Endocrinol 2001;145 (02):137-145

116 Fleseriu M, Hoffman AR, Katznelson L; AACE Neuroendocrine and Pituitary Scientific Committee. Pituitary scientific C: American Association of Clinical Endocrinologists and American College of Endocrinology Disease State clinical review: management of acromegaly patients: what is the role of pre-operative medical therapy? Endocr Pract 2015;21(06):668-673

117 Katznelson L, Atkinson JL, Cook DM, Ezzat SZ, Hamrahian AH, Miller KK; American Association of Clinical Endocrinologists. American Association of Clinical Endocrinologists medical guidelines for clinical practice for the diagnosis and treatment of acromegaly-2011 update. Endocr Pract 2011;17(Suppl 4):1-44

118 Carlsen SM, Lund-Johansen M, Schreiner T, et al; Preoperative Octreotide Treatment of Acromegaly study group. Preoperative octreotide treatment in newly diagnosed acromegalic patients with macroadenomas increases cure short-term postoperative rates: a prospective, randomized trial. J Clin Endocrinol Metab 2008;93(08):2984-2990

119 Losa M, Mortini P, Urbaz L, Ribotto P, Castrignanó T, Giovanelli M. Presurgical treatment with somatostatin analogs in patients with acromegaly: effects on the remission and complication rates. J Neurosurg 2006;104(06):899-906

120 Mao ZG, Zhu YH, Tang HL, et al. Preoperative lanreotide treatment in acromegalic patients with macroadenomas increases short-term postoperative cure rates: a prospective, randomised trial. Eur J Endocrinol 2010;162(04):661-666

121 Laws ER, Scheithauer BW, Groover RV. Pituitary adenomas in childhood and adolescence. Prog Exp Tumor Res 1987;30: 359-361

122 Tarapore PE, Sughrue ME, Blevins L, Auguste KI, Gupta N, Kunwar S. Microscopic endonasal transsphenoidal pituitary adenomectomy in the pediatric population. J Neurosurg Pediatr 2011;7(05): 501-509

123 Fujioka M, Young LW. The sphenoidal sinuses: radiographic patterns of normal development and abnormal findings in infants and children. Radiology 1978;129(01):133

124 Jang YJ, Kim SC. Pneumatization of the sphenoid sinus in children evaluated by magnetic resonance imaging. Am J Rhinol 2000;14 (03):181-185
125 Szolar D, Preidler K, Ranner G, et al. Magnetic resonance assessment of age-related development of the sphenoid sinus. Br J Radiol 1994;67(797):431-435

126 Locatelli D, Massimi L, Rigante M, et al. Endoscopic endonasal transsphenoidal surgery for sellar tumors in children. Int J Pediatr Otorhinolaryngol 2010;74(11):1298-1302

127 Banu MA, Rathman A, Patel KS, et al. Corridor-based endonasal endoscopic surgery for pediatric skull base pathology with detailed radioanatomic measurements. Neurosurgery 2014;10 (Suppl 2):273-293, discussion 293

128 Tatreau JR, Patel MR, Shah RN, et al. Anatomical considerations for endoscopic endonasal skull base surgery in pediatric patients. Laryngoscope 2010;120(09):1730-1737

129 Shah RN, Surowitz JB, Patel MR, et al. Endoscopic pedicled nasoseptal flap reconstruction for pediatric skull base defects. Laryngoscope 2009;119(06):1067-1075

130 Kanter AS, Diallo AO, Jane JA Jr, et al. Single-center experience with pediatric Cushing's disease. J Neurosurg 2005;103(5, Suppl) 413-420

131 Berker M, Hazer DB, Yücel T, et al. Complications of endoscopic surgery of the pituitary adenomas: analysis of 570 patients and review of the literature. Pituitary 2012;15(03):288-300

132 Bokhari AR, Davies MA, Diamond T. Endoscopic transsphenoidal pituitary surgery: a single surgeon experience and the learning curve. Br J Neurosurg 2013;27(01):44-49

133 Cappabianca P, Alfieri A, de Divitiis E. Endoscopic endonasal transsphenoidal approach to the sella: towards functional endoscopic pituitary surgery (FEPS). Minim Invasive Neurosurg 1998; 41(02):66-73

134 Charalampaki P, Ayyad A, Kockro RA, Perneczky A. Surgical complications after endoscopic transsphenoidal pituitary surgery. J Clin Neurosci 2009;16(06):786-789

135 Halvorsen H, Ramm-Pettersen J, Josefsen R, et al. Surgical complications after transsphenoidal microscopic and endoscopic surgery for pituitary adenoma: a consecutive series of 506 procedures. Acta Neurochir (Wien) 2014;156(03):441-449

136 Kassam AB, Prevedello DM, Carrau RL, et al. Endoscopic endonasal skull base surgery: analysis of complications in the authors' initial 800 patients. J Neurosurg 2011;114(06):1544-1568

137 Prevedello DM, Doglietto F, Jane JA Jr, Jagannathan J, Han J, Laws ER Jr. History of endoscopic skull base surgery: its evolution and current reality. J Neurosurg 2007;107(01):206-213

138 Yano S, Hide T, Shinojima N. Efficacy and complications of endoscopic skull base surgery for giant pituitary adenomas. World Neurosurg 2017;99:533-542

139 Bastos RV, Silva CM, Tagliarini JV, et al. Endoscopic versus microscopic transsphenoidal surgery in the treatment of pituitary tumors: systematic review and meta-analysis of randomized and non-randomized controlled trials. Arch Endocrinol Metab 2016;60(05):411-419

140 Cho DY, Liau WR. Comparison of endonasal endoscopic surgery and sublabial microsurgery for prolactinomas. Surg Neurol 2002;58(06):371-375, discussion 375-376

141 Enseñat J, Quesada JL, Aparicio J, et al. Comparación del abordje transesfenoidal microquirúrgico frente al abordaje endonasal transesfenoidal endosócpico. Estudio prospectivo de 50 pacientes. Neurocirugia (Astur) 2009;20(04):335-344, discussion 344-345

142 Jain AK, Gupta AK, Pathak A, Bhansali A, Bapuraj JR. Excision of pituitary adenomas: randomized comparison of surgical modalities. Br J Neurosurg 2007;21(04):328-331

143 Kahilogullari G, Beton S, Al-Beyati ES, et al. Olfactory functions after transsphenoidal pituitary surgery: endoscopic versus microscopic approach. Laryngoscope 2013;123(09):2112-2119

144 Little AS, Chapple K, Jahnke H, White WL. Comparative inpatient resource utilization for patients undergoing endoscopic or microscopic transsphenoidal surgery for pituitary lesions. J Neurosurg 2014;121(01):84-90 
145 Storr HL, Drake WM, Evanson J, et al. Endonasal endoscopic transsphenoidal pituitary surgery: early experience and outcome in paediatric Cushing's disease. Clin Endocrinol (Oxf) 2014;80(02):270-276

146 Massimi L, Rigante M, D’Angelo L, et al. Quality of postoperative course in children: endoscopic endonasal surgery versus sublabial microsurgery. Acta Neurochir (Wien) 2011;153(04): 843-849

147 Rigante M, Massimi L, Parrilla C, et al. Endoscopic transsphenoidal approach versus microscopic approach in children. Int J Pediatr Otorhinolaryngol 2011;75(09):1132-1136

148 Chivukula S, Koutourousiou M, Snyderman CH, Fernandez-Miranda JC, Gardner PA, Tyler-Kabara EC. Endoscopic endonasal skull base surgery in the pediatric population. J Neurosurg Pediatr 2013;11(03):227-241

149 Zhan R, Xu G, Wiebe TM, Li X. Surgical outcomes of the endoscopic transsphenoidal route to pituitary tumours in paediatric patients $>10$ years of age: 5 years of experience at a single institute. Arch Dis Child 2015;100(08):774-778

150 Snyderman $\mathrm{CH}$, Pant H, Carrau RL, Prevedello D, Gardner P, Kassam $\mathrm{AB}$. What are the limits of endoscopic sinus surgery?: the expanded endonasal approach to the skull base Keio J Med 2009;58(03):152-160

151 AlQahtani A, Turri-Zanoni M, Dallan I, Battaglia P, Castelnuovo P. Endoscopic endonasal resection of sinonasal and skull base malignancies in children: feasibility and outcomes. Childs Nerv Syst 2012;28(11):1905-1910

152 D'Haens J, Van Rompaey K, Stadnik T, Haentjens P, Poppe K, Velkeniers B. Fully endoscopic transsphenoidal surgery for functioning pituitary adenomas: a retrospective comparison with traditional transsphenoidal microsurgery in the same institution. Surg Neurol 2009;72(04):336-340

153 Dehdashti AR, Ganna A, Karabatsou K, Gentili F. Pure endoscopic endonasal approach for pituitary adenomas: early surgical results in 200 patients and comparison with previous microsurgical series. Neurosurgery 2008;62(05):1006-1015, discussion 1015-1017

154 Graffeo CS, Dietrich AR, Grobelny B, et al. A panoramic view of the skull base: systematic review of open and endoscopic endonasal approaches to four tumors. Pituitary 2014;17(04): 349-356

155 Higgins TS, Courtemanche C, Karakla D, et al. Analysis of transnasal endoscopic versus transseptal microscopic approach for excision of pituitary tumors. Am J Rhinol 2008;22(06):649-652

156 O'Malley BW Jr, Grady MS, Gabel BC, et al. Comparison of endoscopic and microscopic removal of pituitary adenomas: single-surgeon experience and the learning curve. Neurosurg Focus 2008;25(06):E10

157 Buchfelder M, Kreutzer J. Transcranial surgery for pituitary adenomas. Pituitary 2008;11(04):375-384

158 Dolenc VV. Transcranial epidural approach to pituitary tumors extending beyond the sella. Neurosurgery 1997;41(03):542-550 , discussion 551-552

159 Couldwell WT. Transsphenoidal and transcranial surgery for pituitary adenomas. J Neurooncol 2004;69(1-3):237-256

160 Jane JA, Park TS, Pobereskin LH, Winn HR, Butler AB. The supraorbital approach: technical note. Neurosurgery 1982;11 (04):537-542

161 Iwai Y, Hakuba A, Katsuyama J, et al. A case of ectopic large pituitary adenoma. No Shinkei Geka 1990;18(01):71-75

162 Ohata K, Takami T, Goto T, Hara M. Transpetrosal look-up approach for retrochiasmatic suprasellar tumors. Skull Base 2005;15:B-9

163 DeLellis RA. Pathology and Genetics of Tumours of Endocrine Organs. vol 8. Lyon, France: IARC; 2004

164 Figarella-Branger D, Trouillas J. The new WHO classification of human pituitary tumors: comments. Acta Neuropathol 2006; 111(01):71-72
165 Saeger W, Honegger J, Theodoropoulou M, et al. Clinical impact of the current WHO classification of pituitary adenomas. Endocr Pathol 2016;27(02):104-114

166 Scheithauer BW, Gaffey TA, Lloyd RV, et al. Pathobiology of pituitary adenomas and carcinomas. Neurosurgery 2006;59 (02):341-353, discussion 341-353

167 George DH, Scheithauer BW, Kovacs K, et al. Crooke's cell adenoma of the pituitary: an aggressive variant of corticotroph adenoma. Am J Surg Pathol 2003;27(10):1330-1336

168 Pernicone PJ, Scheithauer BW, Sebo TJ, et al. Pituitary carcinoma: a clinicopathologic study of 15 cases. Cancer 1997;79(04): 804-812

169 Thapar K, Yamada Y, Scheithauer B, Kovacs K, Yamada S, Stefaneanu L. Assessment of mitotic activity in pituitary adenomas and carcinomas. Endocr Pathol 1996;7(03):215-221

170 Di leva A, Rotondo F, Syro LV, Cusimano MD, Kovacs K. Aggressive pituitary adenomas-diagnosis and emerging treatments. Nat Rev Endocrinol 2014;10(07):423-435

171 Zada G, Woodmansee WW, Ramkissoon S, Amadio J, Nose V Laws ER Jr. Atypical pituitary adenomas: incidence, clinical characteristics, and implications. J Neurosurg 2011;114(02): 336-344

172 Ram Z, Nieman LK, Cutler GB Jr, Chrousos GP, Doppman JL, Oldfield EH. Early repeat surgery for persistent Cushing's disease. J Neurosurg 1994;80(01):37-45

173 Landolt AM, Haller D, Lomax N, et al. Octreotide may act as a radioprotective agent in acromegaly. J Clin Endocrinol Metab 2000;85(03):1287-1289

174 Landolt AM, Haller D, Lomax N, et al. Stereotactic radiosurgery for recurrent surgically treated acromegaly: comparison with fractionated radiotherapy. J Neurosurg 1998;88(06):1002-1008

175 Pollock BE, Nippoldt TB, Stafford SL, Foote RL, Abboud CF. Results of stereotactic radiosurgery in patients with hormone-producing pituitary adenomas: factors associated with endocrine normalization. J Neurosurg 2002;97(03):525-530

176 Goffman TE, Dewan R, Arakaki R, Gorden P, Oldfield EH, Glatstein E. Persistent or recurrent acromegaly. Long-term endocrinologic efficacy and neurologic safety of postsurgical radiation therapy. Cancer 1992;69(01):271-275

177 Pollock BE, Carpenter PC. Stereotactic radiosurgery as an alternative to fractionated radiotherapy for patients with recurrent or residual nonfunctioning pituitary adenomas. Neurosurgery 2003;53(05):1086-1091, discussion 1091-1094

178 Rutkowski MJ, Flanigan PM, Aghi MK. Update on the management of recurrent Cushing's disease. Neurosurg Focus 2015;38 (02):E16

179 Sheehan JM, Vance ML, Sheehan JP, Ellegala DB, Laws ER Jr. Radiosurgery for Cushing's disease after failed transsphenoidal surgery. J Neurosurg 2000;93(05):738-742

180 Sheehan JP, Kondziolka D, Flickinger J, Lunsford LD. Radiosurgery for residual or recurrent nonfunctioning pituitary adenoma. J Neurosurg 2002;97(5, Suppl):408-414

181 Loeffler JS, Shih HA. Radiation therapy in the management of pituitary adenomas. J Clin Endocrinol Metab 2011;96(07): 1992-2003

182 Sheehan JP, Xu Z, Lobo MJ. External beam radiation therapy and stereotactic radiosurgery for pituitary adenomas. Neurosurg Clin N Am 2012;23(04):571-586

183 Thorén M, Rähn T, Hallengren B, et al. Treatment of Cushing's disease in childhood and adolescence by stereotactic pituitary irradiation. Acta Paediatr Scand 1986;75(03):388-395

184 Devoe DJ, Miller WL, Conte FA, et al. Long-term outcome in children and adolescents after transsphenoidal surgery for Cushing's disease. J Clin Endocrinol Metab 1997;82(10):3196-3202

185 Magiakou MA, Mastorakos G, Oldfield EH, Gomez MT, Doppman JL, Cutler GB Jr, et al. Cushing's Syndrome in Children and Adolescents-Presentation, Diagnosis, and Therapy. N Engl J Med 1994;331:629-636 
186 Ding D, Starke RM, Sheehan JP. Treatment paradigms for pituitary adenomas: defining the roles of radiosurgery and radiation therapy. J Neurooncol 2014;117(03):445-457

187 Estrada J, Boronat M, Mielgo M, et al. The long-term outcome of pituitary irradiation after unsuccessful transsphenoidal surgery in Cushing's disease. N Engl J Med 1997;336(03):172-177

188 Jennings AS, Liddle GW, Orth DN. Results of treating childhood Cushing's disease with pituitary irradiation. N Engl J Med 1977; 297(18):957-962

189 Petit JH, Biller BM, Yock TI, et al. Proton stereotactic radiotherapy for persistent adrenocorticotropin-producing adenomas. J Clin Endocrinol Metab 2008;93(02):393-399

190 Ronson BB, Schulte RW, Han KP, Loredo LN, Slater JM, Slater JD. Fractionated proton beam irradiation of pituitary adenomas. Int J Radiat Oncol Biol Phys 2006;64(02):425-434

191 Graffeo CS, Perry A, Carlstrom LP, et al. Characterizing and predicting the Nelson-Salassa syndrome. J Neurosurg 2017; 127(06):1277-1287

192 Pollock BE, Young WF Jr. Stereotactic radiosurgery for patients with ACTH-producing pituitary adenomas after prior adrenalectomy. Int J Radiat Oncol Biol Phys 2002;54(03):839-841

193 Heaney AP. Clinical review: Pituitary carcinoma: difficult diagnosis and treatment. J Clin Endocrinol Metab 2011;96(12): 3649-3660

194 Losa M, Bogazzi F, Cannavo S, et al. Temozolomide therapy in patients with aggressive pituitary adenomas or carcinomas. J Neurooncol 2016;126(03):519-525

195 McCormack AI, Wass JA, Grossman AB. Aggressive pituitary tumours: the role of temozolomide and the assessment of MGMT status. Eur J Clin Invest 2011;41(10):1133-1148

196 Ortiz LD, Syro LV, Scheithauer BW, et al. Temozolomide in aggressive pituitary adenomas and carcinomas. Clinics (Sao Paulo) 2012;67(Suppl 1):119-123

197 Raverot G, Castinetti F, Jouanneau E, et al. Pituitary carcinomas and aggressive pituitary tumours: merits and pitfalls of temozolomide treatment. Clin Endocrinol (Oxf) 2012;76(06): 769-775

198 Fukuoka H, Cooper O, Ben-Shlomo A, et al. EGFR as a therapeutic target for human, canine, and mouse ACTH-secreting pituitary adenomas. J Clin Invest 2011;121(12):4712-4721

199 Fukuoka H, Cooper O, Mizutani J, et al. HER2/ErbB2 receptor signaling in rat and human prolactinoma cells: strategy for targeted prolactinoma therapy. Mol Endocrinol 2011;25(01): 92-103

200 Jouanneau E, Wierinckx A, Ducray F, et al. New targeted therapies in pituitary carcinoma resistant to temozolomide. Pituitary 2012;15(01):37-43

201 Ortiz LD, Syro LV, Scheithauer BW, et al. Anti-VEGF therapy in pituitary carcinoma. Pituitary 2012;15(03):445-449
202 Katznelson L, Klibanski A. Prolactinomas. Cancer Treat Res 1997; 89:41-55

203 Rasmussen C. Hyperprolactinaemia-a clinical study with special reference to long-term follow-up, treatment with dopamine agonists, and pregnancy. Ups J Med Sci 1990;95(01):1-29

204 Rasmussen C, Bergh T, Nillius SJ, Wide L. Return of menstruation and normalization of prolactin in hyperprolactinemic women with bromocriptine-induced pregnancy. Fertil Steril 1985;44 (01):31-34

205 Gsponer J, De Tribolet N, Déruaz JP, et al. Diagnosis, treatment, and outcome of pituitary tumors and other abnormal intrasellar masses. Retrospective analysis of 353 patients. Medicine (Baltimore) 1999;78(04):236-269

206 Hoorn EJ, Zietse R. Water balance disorders after neurosurgery: the triphasic response revisited. NDT Plus 2010;3(01):42-44

207 Das NK, Lyngdoh BT, Bhakri BK, et al. Surgical management of pediatric Cushing's disease. Surg Neurol 2007;67(03):251-257, discussion 257

208 Kassam A, Thomas AJ, Snyderman C, et al. Fully endoscopic expanded endonasal approach treating skull base lesions in pediatric patients. J Neurosurg 2007;1062, Suppl):75-86

209 Tamasauskas A, Sinkūnas K, Draf W, et al. Management of cerebrospinal fluid leak after surgical removal of pituitary adenomas. Medicina (Kaunas) 2008;44(04):302-307

210 Garcia-Navarro V, Anand VK, Schwartz TH. Gasket seal closure for extended endonasal endoscopic skull base surgery: efficacy in a large case series. World Neurosurg 2013;80(05):563-568

211 Kassam AB, Thomas A, Carrau RL, et al. Endoscopic reconstruction of the cranial base using a pedicled nasoseptal flap. Neurosurgery 2008;63(01, Suppl 1):ONS44-ONS52, discussion ONS52-ONS53

212 Richmond IL, Wilson CB. Pituitary adenomas in childhood and adolescence. J Neurosurg 1978;49:163-168

213 Fahlbusch R, Buchfelder M, Muller OA. Transsphenoidal surgery for Cushing's disease. J R Soc Med 1986;79:262-269

214 Lüdecke D, Herrmann H-D, Schulte F. Special problems with neurosurgical treatment of hormone-secreting pituitary adenomas in children. In: Kageyama N, Takakura K, Epstein FJ, Hoffman HJ, Schut L, eds. Intracranial Tumors in Infancy and Childhood Vol 30. New York, NY: Karger Publishers; 1987:362-370

215 Haddad SF, VanGilder JC, Menezes AH. Pediatric pituitary tumors. Neurosurgery 1991;29:509-514

216 Leinung MC, Kane LA, Scheithauer BW, Carpenter PC, Laws ER Jr., Zimmerman D. Long term follow-up of transsphenoidal surgery for the treatment of Cushing's disease in childhood. J Clin Endocrinol Metab 1995;80:2475-2479

217 Abe T, Ludecke DK, Saeger W. Clinically nonsecreting pituitary adenomas in childhood and adolescence. Neurosurgery 1998; 42:744-750; discussion 750-741 